\title{
Novel Variance-Component TWAS method for studying complex human diseases with applications to Alzheimer's dementia
}

\author{
Shizhen Tang $\circledast^{1,2}$, Aron S. Buchman $\oplus^{3}$, Philip L. De Jager $\oplus^{4}$, David A. Bennett ${ }^{3}$, Michael \\ P. Epstein ${ }^{1}$, Jingjing Yang ${ }^{1}$ * \\ 1 Center for Computational and Quantitative Genetics, Department of Human Genetics, Emory University \\ School of Medicine, Atlanta, Georgia, United States of America, 2 Department of Biostatistics and \\ Bioinformatics, Emory University School of Public Health, Atlanta, Georgia, United States of America, 3 Rush \\ Alzheimer's Disease Center, Rush University Medical Center, Chicago, Illinois, United States of America, \\ 4 Center for Translational and Computational Neuroimmunology, Department of Neurology and Taub \\ Institute for Research on Alzheimer's Disease and the Aging Brain, Columbia University Irving Medical \\ Center, New York, New York, United States of America \\ * jingjing.yang@emory.edu
}

\section{OPEnACCESS}

Citation: Tang S, Buchman AS, De Jager PL, Bennett DA, Epstein MP, Yang J (2021) Novel Variance-Component TWAS method for studying complex human diseases with applications to Alzheimer's dementia. PLoS Genet 17(4): e1009482. https://doi.org/10.1371/journal. pgen.1009482

Editor: Lin Chen, The University of Chicago, UNITED STATES

Received: June 26, 2020

Accepted: March 15, 2021

Published: April 2, 2021

Peer Review History: PLOS recognizes the benefits of transparency in the peer review process; therefore, we enable the publication of all of the content of peer review and author responses alongside final, published articles. The editorial history of this article is available here: https://doi.org/10.1371/journal.pgen.1009482

Copyright: @ 2021 Tang et al. This is an open access article distributed under the terms of the Creative Commons Attribution License, which permits unrestricted use, distribution, and reproduction in any medium, provided the original author and source are credited.

Data Availability Statement: All data used in this paper are available either from public website or requested from the following websites. RADC

\section{Abstract}

Transcriptome-wide association studies (TWAS) have been widely used to integrate transcriptomic and genetic data to study complex human diseases. Within a test dataset lacking transcriptomic data, traditional two-stage TWAS methods first impute gene expression by creating a weighted sum that aggregates SNPs with their corresponding cis-eQTL effects on reference transcriptome. Traditional TWAS methods then employ a linear regression model to assess the association between imputed gene expression and test phenotype, thereby assuming the effect of a cis-eQTL SNP on test phenotype is a linear function of the eQTL's estimated effect on reference transcriptome. To increase TWAS robustness to this assumption, we propose a novel Variance-Component TWAS procedure (VC-TWAS) that assumes the effects of cis-eQTL SNPs on phenotype are random (with variance proportional to corresponding reference cis-eQTL effects) rather than fixed. VC-TWAS is applicable to both continuous and dichotomous phenotypes, as well as individual-level and summary-level GWAS data. Using simulated data, we show VC-TWAS is more powerful than traditional TWAS methods based on a two-stage Burden test, especially when eQTL genetic effects on test phenotype are no longer a linear function of their eQTL genetic effects on reference transcriptome. We further applied VC-TWAS to both individual-level (N $=\sim 3.4 \mathrm{~K})$ and summary-level $(\mathrm{N}=\sim 54 \mathrm{~K})$ GWAS data to study Alzheimer's dementia (AD). With the individual-level data, we detected 13 significant risk genes including 6 known GWAS risk genes such as TOMM4O that were missed by traditional TWAS methods. With the summary-level data, we detected 57 significant risk genes considering only cis-SNPs and 71 significant genes considering both cis- and trans- SNPs, which also validated our findings with the individual-level GWAS data. Our VC-TWAS method is implemented in the TIGAR tool for public use. 
Research Resource Sharing Hub, http://www.radc. rush.edu/; ROS/MAP data, https://www.synapse. org/\#!Synapse:syn3219045; MayoClinicLOAD data, https://www.synapse.org/\#!Synapse: syn2910256; IGAP summary data, https://web. pasteur-lille.fr/en/recherche/u744/igap/igap_ download.php.

Funding: ST and JY are supported by National Institutes of Health (NIH/NIGMS) grant award R35GM138313. MPE was supported by NIH/ NIGMS grant award R01GM117946 and NIH/NIA grant award RF1AG071170. Data collection was supported through funding by NIH/NIA grants P30AG10161, R01AG15819, R01AG17917, R01AG30146, R01AG36836, R01AG56352, U01AG32984, U01AG46152, U01AG61356, the Illinois Department of Public Health, and the Translational Genomics Research Institute. These grants support the generation of the ROS/MAP data, which is led by ASB, PLDJ and DAB. The funders had no role in study design, data collection and analysis, decision to publish, or preparation of the manuscript.

Competing interests: The authors have declared that no competing interests exist.

\section{Author summary}

Traditional Transcriptome-wide association studies (TWAS) tools make strong assumptions about the relationships among genetic variants, transcriptome, and phenotype that may be violated in practice, thereby substantially reducing the power. Here, we propose a Variance-Component TWAS method (VC-TWAS) that relaxes these assumptions and can be implemented with both individual-level and summary-level GWAS data, which is suitable for studying both continuous and dichotomous phenotypes. Our simulation studies showed that VC-TWAS achieved higher power compared to traditional TWAS methods based on a two-stage Burden test, when the underlying assumptions required by traditional TWAS tools were violated. We further applied VC-TWAS to both individuallevel $(\mathrm{N}=\sim 3.4 \mathrm{~K})$ and summary-level $(\mathrm{N}=\sim 54 \mathrm{~K})$ GWAS data to study Alzheimer's dementia (AD). With individual-level data, we detected 13 significant risk genes including 6 known GWAS risk genes such as TOMM40 that were missed by traditional TWAS methods. Interestingly, 5 of these genes were shown to possess significant pleiotropic effects on $\mathrm{AD}$ pathology phenotypes, revealing possible biological mechanisms. With summary-level data of a larger sample size, we detected 57 significant risk genes considering only cis-SNPs and 71 significant genes considering both cis- and trans- SNPs, which also validated our findings with the individual-level GWAS data. In conclusion, VCTWAS provides an important analytic tool for identifying risk genes whose effects on phenotypes might be mediated through transcriptomes.

\section{Introduction}

Genome-wide association studies (GWAS) have succeeded in identifying thousands of genetic loci associated with complex traits and diseases [1-3]. However, for the most part the molecular mechanisms linking these genes with these complex traits and diseases remain unexplained [4]. Studies have shown that gene expression plays a key role in the phenotypic manifestation of human diseases [5]. Many common genetic variants associated with the phenotypes manifested by human diseases are highly likely to be expression quantitative trait loci (eQTL) [6,7]. Therefore, integrating gene expression data together with genetic data from GWAS is expected to help identify novel risk genes as well as elucidate the mechanisms underlying the associations of genetic loci with complex traits and disease phenotypes.

The transcriptome-wide association study (TWAS) is an innovative strategy to leverage the enhanced power of integrating gene expression of the transcriptome together with genetic data from GWAS [8] for gene-based association studies of complex traits. Using a reference transcriptomic panel like Genotype-Tissues Expression (GTEx) [9], traditional TWAS methods first train a regression model that treat gene expression as the outcome and SNP genotype data (generally cis-SNPs nearby the test gene) as predictors, which can be viewed as a gene expression imputation model. traditional two-stage TWAS tools such as PrediXcan [10], FUSION [11], and TIGAR [8] employ different regression methods to fit such gene expression imputation models, where corresponding cis-eQTL effect sizes are derived. For example, PrediXcan method [10] implements the Elastic-Net penalized regression method [12] while TIGAR [8] implements the nonparametric Bayesian Dirichlet process regression (DPR) [13] method. Regardless of technique, these existing two-stage TWAS methods produce a set of estimates of cis-eQTL effects on reference transcriptome. Within a GWAS lacking transcriptomic data, existing two-stage TWAS tools then imputes genetically-regulated gene expression 
(GReX) by weighted summing cis-eQTL SNP data from the GWAS with corresponding estimates of cis-eQTL effects on reference transcriptome. Once created, existing two-stage TWAS tools test for association between the imputed GReX and traits of interest using GWAS data, where the test is based on a single variant linear regression model. TWAS methods have been deployed widely since their inception and have had substantial success in improving our understanding the genetic regulation of various complex traits [14].

Since existing two-stage TWAS tools derive GReX as the summation of eQTL SNP genotypes weighted by their corresponding effects on reference transcriptome, they essentially conduct weighted burden tests [8] to evaluate the association between GReX and outcome (referred to as Burden-TWAS in this paper). Burden-TWAS methods assume the effects of eQTL SNPs on phenotype is a linear function of their corresponding estimated effects on reference transcriptome (see Methods), which may not be true in practice. In particular, the effect of eQTL SNP on phenotype may be a non-linear, rather than a linear, function of the eQTL effect on reference transcriptome. Moreover, the assumed eQTL effects on transcriptome in the test dataset could be mis-specified due to ancestral differences between the test and reference datasets. For example, recent studies show that gene-expression prediction models trained in one population perform poorly when applied to a different population [15]. The non-linearity assumption about the effects of eQTL SNPs on the phenotype of interest does not affect the validity of TWAS but it can reduce the power of these traditional two-stage Burden-TWAS methods.

Here, we derive a Variance-Component TWAS (VC-TWAS) method that relaxes the linearity assumption of Burden-TWAS. This modification makes this method more robust to misspecification of eQTL effect size estimates derived from the reference transcriptome, thus improving its power compared to the Burden-TWAS methods. VC-TWAS is a TWAS analogue of the Sequence Kernel Association Test (SKAT) [16-19] used for gene-based association studies. Unlike Burden-TWAS methods, our VC-TWAS aggregates genetic information across test SNPs using a kernel similarity function that allows upweighting or downweighing of specific variants in the similarity score based on eQTL effect size magnitudes (which can be derived using DPR methods in TIGAR or Elastic-Net penalized regression methods implemented in PrediXcan). The test statistic can be thought of as a Variance-Component score statistic based on a mixed model where each test variant has a random effect whose variance is a linear function of the squared values of corresponding eQTL effect size.

By modeling variants with random effects, the technique relaxes the main assumption of Burden-TWAS and is robust to misspecification of eQTL effect size estimates derived from the reference transcriptome (in terms of the direction and magnitude). The variance component score test employed by VC-TWAS also enjoys efficient computation and flexibility for studying both quantitative and dichotomous phenotypes, which enables VC-TWAS to consider both cis- and trans- eQTL SNPs. VC-TWAS is further applicable to both individual-level and summary-level GWAS data.

We note that VC-TWAS is not the first TWAS method developed that relaxes the linearity assumption of Burden-TWAS. A recently derived collaborative mixed model (CoMM) [20,21] likewise accounts for the uncertainty of eQTL effect size estimates from reference transcriptome data by jointly modeling reference and test data within a linear mixed-model framework. The PMR-Egger [22] and moPMR-Egger [23] also take the approach of jointly modeling reference and test data to address the uncertainty of eQTL effect size estimates from the reference data. However, these methods that are based on the maximum likelihood inference framework and implement likelihood ratio tests, are derived only for quantitative phenotypes and could suffer computation burden for testing thousands of cis-SNPs per gene, which often happens in practice particularly when considering imputed SNP data or whole-genome sequencing data for TWAS. In this study, we show that the likelihood ratio test approach used by CoMM and 
PMR-Egger is computationally more expensive to run than VC-TWAS in practice when there are thousands of test SNPs such as using imputed or Whole Genome Sequencing (WGS) genotype data.

This manuscript is as organized as follows. First, we provide an overview of traditional twostage Burden-TWAS as well as existing techniques for estimating cis-eQTL SNP effects from reference transcriptome data. We then derive VC-TWAS approach for using both individuallevel and summary-level GWAS data and compare its performance with Burden-TWAS using simulated data generated under different eQTL model assumptions. We then apply VC-TWAS to individual-level GWAS data from Religious Order Study and Memory Aging Project (ROS/ MAP) [24-27] and Mayo Clinic Late-Onsite Alzheimer's disease (LOAD) [28,29] cohorts, as well as the summary-level GWAS data from the International Genomics of Alzheimer's Project (IGAP) [3], for studying Alzheimer's dementia (AD). By considering only cis-eQTL effect sizes, VC-TWAS identified both novel and known risk genes for AD within $2 \mathrm{MB}$ of the wellknown major risk gene $A P O E$ of $\mathrm{AD}$, including the known risk gene TOMM40 and APOE. Considering both cis- and trans- eQTL effect sizes estimated by the Bayesian Genome-wide TWAS (BGW-TWAS) method [30], VC-TWAS detected 71 risk genes for AD which complemented existing TWAS results using only cis-eQTL SNP data. After describing the results, we provide a brief discussion summarizing our findings and describing implementation of VC-TWAS into our previously developed software tool TIGAR [8] for public use.

\section{Methods}

\section{Ethics statement}

Real ROS/MAP and Mayo Clinic GWAS data analyzed in this study were generated under the improvement by the Institutional Review Board (IRB) of Rush University Medical Center, Chicago, IL and Mayo Clinic, respectively. All samples analyzed in this study were de-identified and all analyses were approved by the IRB of Emory University School of Medicine.

\section{Traditional Two-Stage TWAS procedure}

Two-stage TWAS first fits gene expression imputation models by taking genotype data as predictors and assuming the following additive genetic model for expression quantitative traits,

$$
\boldsymbol{E}_{g}=\boldsymbol{G} \boldsymbol{w}+\boldsymbol{\varepsilon}, \boldsymbol{\varepsilon} \sim N\left(0, \sigma_{\epsilon}^{2} \boldsymbol{I}\right) .
$$

Here, $\boldsymbol{G}$ is the genotype matrix for all considered SNP genotypes (encoded as the number of minor alleles or genotype dosages of SNPs within $1 \mathrm{MB}$ of the target gene region), $w$ is the eQTL effect size vector, and $\boldsymbol{E}_{\boldsymbol{g}}$ is the profiled gene expression levels for the target gene $\boldsymbol{g}$. Given the eQTL effect size estimates $\widehat{\boldsymbol{w}}$ from reference data, $\mathbf{G R e X}$ will be imputed by the following equation

$$
\widehat{G R e X}=G_{n e w} \widehat{w},
$$

where $\boldsymbol{G}_{\text {new }}$ is the genotype matrix for the test cohort.

The general test framework of Burden-TWAS $[8,10,11]$ that test for association between

$\widehat{G R \boldsymbol{X}}$ and the phenotype of interest can be written as:

$$
\boldsymbol{E}\left[\boldsymbol{g}\left(\boldsymbol{Y} \mid \boldsymbol{G}_{\text {new }}\right)\right]=\beta \widehat{\boldsymbol{G R e} \boldsymbol{X}}+\boldsymbol{\alpha} \mathbf{Z}=\beta\left(\boldsymbol{G}_{\text {new }} \widehat{\boldsymbol{w}}\right)+\boldsymbol{\alpha} \mathbf{Z},
$$

where $\boldsymbol{Y}$ denotes the phenotype of interest, $g($.) denotes a link function, $\widehat{\boldsymbol{G R e} X}$ is imputed gene expression levels, and $\boldsymbol{\alpha}^{\prime}$ denotes the coefficient vector for other non-genomic covariates $\boldsymbol{Z}$. 
Basically, Burden-TWAS tests the null hypothesis of $H_{0}: \beta=0$, where eQTL effect size estimates $(\widehat{\boldsymbol{w}})$ are taken as variant weights and SNP effect sizes on phenotype $(\beta \widehat{\boldsymbol{w}})$ are assumed to be a linear function of $\widehat{\boldsymbol{w}}[8,10,11]$. As noted above this linear relationship between eQTL effect sizes on reference transcriptome and the SNP effect sizes on test phenotype may not be true when analyzing human data and limits the power of the existing two-stage Burden-TWAS methods.

\section{Estimation of eQTL effect sizes}

Different methods can be used to estimate eQTL effect sizes $\boldsymbol{w}$ from Eq (1). In this study, we applied PrediXcan (Elastic-Net penalized regression) and TIGAR (nonparametric Bayesian DPR methods) $[8,10]$ to estimate $\boldsymbol{w}$ that only consider cis-eQTL. PrediXcan TWAS method [10] employs Elastic-Net penalized regression method [12] to estimate cis-eQTL effect sizes $\boldsymbol{w}$ from Eq (1) (S1A Text). TIGAR ${ }^{8}$ provides a more flexible approach to nonparametrically estimate cis-eQTL effect sizes by a Bayesian DPR method [13] (S1B Text). Additionally, we also considered modeling gene expression using both cis- and trans- eQTL effect sizes estimated by the recently proposed Bayesian genome-wide TWAS (BGW-TWAS) method [30].

\section{VC-TWAS with individual-level GWAS data}

Here, we propose a powerful VC-TWAS method that is analogous to the previously proposed SKAT method for SNP-set based association studies [16]. Similar to SKAT, the general test framework of VC-TWAS can be written as

$$
Y_{i}=\boldsymbol{\beta}^{\prime} \boldsymbol{G}_{i}+\boldsymbol{\alpha}^{\prime} Z_{i}+\varepsilon_{i}, \beta_{j}^{\prime} \sim N\left(0, w_{j}^{2} \tau\right), \boldsymbol{\varepsilon}_{i} \sim N\left(0, \sigma_{\epsilon}^{2}\right)
$$

for continuous quantitative traits, and

$$
\operatorname{logit}\left(\operatorname{Prob}\left(Y_{i}=1\right)\right)=\boldsymbol{\beta}^{\prime} \boldsymbol{G}_{i}+\boldsymbol{\alpha}^{\prime} \mathbf{Z}_{\boldsymbol{i}}+\boldsymbol{\varepsilon}_{i}, \beta_{j}^{\prime} \sim N\left(0, w_{j}^{2} \tau\right), \boldsymbol{\varepsilon}_{i} \sim N\left(0, \sigma_{\epsilon}^{2}\right),
$$

for dichotomous traits of sample $i$. Here, $\boldsymbol{\beta}$ is the genetic effect size vector, $\boldsymbol{G}$ is the genotype matrix for all test SNPs with respect to the test gene, $\boldsymbol{Z}$ is the non-genomic covariate matrix, and $\boldsymbol{\varepsilon}$ is the error term. VC-TWAS will test $H_{0}: \tau=0$, which is equivalent to testing $H_{0}: \boldsymbol{\beta}=0$. The Variance-Component score statistic used by VC-TWAS is given by

$$
Q=(\boldsymbol{Y}-\widehat{\boldsymbol{\mu}})^{\prime} \boldsymbol{K}(\boldsymbol{Y}-\widehat{\boldsymbol{\mu}}), \boldsymbol{K}=\boldsymbol{G} \boldsymbol{W} \boldsymbol{G}^{\prime},
$$

where $\widehat{\boldsymbol{\mu}}$ is the estimated phenotype mean under $\boldsymbol{H}_{\mathbf{0}}$ and $\boldsymbol{W}=\operatorname{diag}\left(w_{j}^{2}, \ldots\right)$ with weight $w_{j}$ for the $j$ th variant.

In contrast to SKAT methods, VC-TWAS takes eQTL effect size estimates from Eq (1) as variant weights $\left(w_{j}\right)$. That is, the variances $\left(\tau w_{j}^{2}\right)$ of SNP effect sizes on phenotype are assumed to be a linear function of eQTL effect size estimates, which is robust to both direction and magnitude of eQTL effect size estimates. Since the Variance-Component score statistic Q (Eq (4)) follows a mixture of chi-square distributions under the null hypothesis [31,32], $\mathrm{p}$-value can be conveniently obtained from several approximation and exact methods like the Davies exact method [33].

We note that our VC-TWAS method is computationally more expensive than standard Burden-TWAS given the need to perform eigen-decomposition of the kernel matrix $\boldsymbol{K}$ in Eq 4 to obtain an analytic p-value (S1C Text). Such eigen-decomposition has computational complexity $\mathrm{O}\left(\mathrm{m}^{3}\right)$ for considering $m$ SNPs with non-zero eQTL effect sizes. As DPR method produces non-zero cis-eQTL effect size estimates for almost all test SNPs (with most cis-eQTL effect size estimates being close to zero [8]), we explored an alternate VC-TWAS that 
considered a reduced set of SNPs by filtering out those with cis-eQTL effect size estimates smaller than the median cis-eQTL effect size estimate per gene. As we show using simulated data, we can reduce up to $80 \%$ computation time while having negligible impact on performance relative to using all SNPs with non-zero cis-eQTL effect size estimates.

Compared to Burden-TWAS, VC-TWAS is based on a random effect model and test for if the variation of random effects differs from 0 . That is, the variance component score statistic (Eq (4)) used by VC-TWAS does not directly model the directions of cis-eQTL effect sizes $\left(w_{j}\right)$ and is robust for mis-specifications of cis-eQTL effect sizes.

\section{VC-TWAS with summary-level GWAS data}

Since summary-level GWAS data generally provide SNP effect sizes on phenotype and corresponding standard errors based on single variant tests (often meta-analysis), it is reasonable to assume that the test phenotypes were adjusted for the confounding covariates. That is, in the $Q$ statistic used by VC-TWAS as in Eq (4), we can assume the phenotype mean $\widehat{\boldsymbol{\mu}}$ under $\boldsymbol{H}_{\mathbf{0}}$ is 0 . This leads to a simplified formula [34,35]

$$
Q=\sum_{j=1}^{m} w_{j}^{2} s_{j}^{2},
$$

where $s_{j}=\boldsymbol{G}_{j .}^{\prime} \mathbf{Y} /{\widehat{\sigma_{Y}}}^{2}$ is the single variant score statistic of the $j^{\text {th }}$ variant, ${\widehat{\sigma_{Y}}}^{2}$ is the estimated phenotype variance.

As derived in the previous studies [36], we can estimate phenotype variance and then the score statistics by using only GWAS summary statistics including the single variant effect size estimate $\widehat{\beta}_{j}$ and corresponding standard error $\widehat{\sigma}_{j}$ for $j^{\text {th }}$ SNP, sample size $n$, and a reference LD covariance matrix $\Sigma$ of all test SNPs (S1C Text). That is, the numerator and denominator of the score statistic can be estimated by,

$$
\boldsymbol{G}_{. j}^{\prime} \boldsymbol{Y}=(n-1) \widehat{\beta}_{j} \Sigma_{j, j} ;{\widehat{\sigma_{Y}}}^{2}=\operatorname{median}\left(\Sigma_{j, j}{\widehat{\sigma_{j}^{2}}}^{2}(n-1)+\Sigma_{j, j} \widehat{\beta}_{j}^{2} ; j=1, \ldots, m\right) .
$$

\section{ROS/MAP data}

In this study we used clinical and postmortem data from older adults participating in two ongoing community-based cohorts studies the Religious Orders Study (ROS) and Rush Memory and Aging Project (MAP) [24-27] which document risk factors and chronic conditions of aging including dementia. Participants are senior adults without known dementia, who agree to annual clinical evaluation and brain autopsy at the time of death. All participants signed an informed consent and Anatomic Gift Act, and the studies are approved by an Institutional Review Board of Rush University Medical Center, Chicago, IL. All participants in this study also signed a repository consent to allow their data to be re-purposed.

Currently, we have microarray genotype data generated for 2,093 European-decent subjects from ROS/MAP [24-27], which are further imputed to the 1000 Genome Project Phase 3 [37] in our analysis [38]. Post-mortem brain samples (gray matter of the dorsolateral prefrontal cortex) from $\sim 30 \%$ these ROS/MAP participants with assayed genotype data are also profiled for transcriptomic data by next-generation RNA-sequencing [39], which are used as reference data to train GReX prediction models in our application studies.

Using ROS/MAP data, we conducted TWAS for clinical diagnosis of late on-site Alzheimer's dementia (LOAD) as well as three pathology indices of $\mathrm{AD}$ including quantified $\beta$-amyloid load and PHFtau tangle density as well as a summary measure of AD pathology burden (S1D Text) $[24,25,27]$. 


\section{Mayo Clinic LOAD GWAS data}

Mayo Clinic LOAD GWAS data contain samples from two clinical AD Case-Control series: Mayo Clinic Jacksonville (MCJ: $353 \mathrm{AD}$ cases and 331 Controls), Mayo Clinic Rochester (MCR: $245 \mathrm{AD}$ cases and 701 Controls) and a neuropathological series of autopsy-confirmed subjects from the Mayo Clinic Brain Bank (MCBB: 246 AD cases and 223 non-AD Controls) $[28,29]$. In total, we have 844 cases with $\mathrm{LOAD}$ and 1,255 controls without a dementia diagnosis. Mayo Clinic LOAD GWAS data have microarray genotype data profiled for 2,099 European-decent samples that are further imputed to the 1000 Genome Project Phase 3 [37] in our analysis [38]. This cohort only profiles the phenotype of clinical diagnosis of AD.

\section{IGAP GWAS summary statistics of AD}

We used the GWAS summary statistics of AD generated from the stage 1 of the International Genomics of Alzheimer's Project (IGAP) with individuals of all European ancestry [3]. This summary-level GWAS data was generated by meta-analysis $(\mathrm{N}=\sim 54 \mathrm{~K})$ of four previouslypublished GWAS datasets consisting of 17,008 Alzheimer's disease cases and 37,154 controls (The European Alzheimer's disease Initiative-EADI the Alzheimer Disease Genetics Consortium-ADGC The Cohorts for Heart and Aging Research in Genomic Epidemiology consortium-CHARGE The Genetic and Environmental Risk in AD consortium-GERAD).

\section{Simulation study design}

The purpose of this simulation study is to compare the performance of Burden-TWAS and VC-TWAS with variant weights estimated by PrediXcan and DPR methods, as well as validate the VC-TWAS approach of using only summary-level GWAS data. We used the real genotype data from ROS/MAP [40] participants to simulate quantitative gene expression and phenotype traits, where the genotype data were of 2,799 cis-SNPs (with $M A F>5 \%$ and Hardy Weinberg p-value $>10^{-5}$ ) of the arbitrarily chosen gene $A B C A 7$.

Specifically, quantitative gene expression traits are generated by the following equation

$$
\boldsymbol{E}_{g}=\boldsymbol{G} \boldsymbol{w}+\boldsymbol{\varepsilon}_{\boldsymbol{E}}
$$

where $\boldsymbol{G}$ denotes the genotype matrix of randomly selected true causal eQTL based on a target proportion of causal eQTL ( $\left.p_{\text {causal }}\right)$ within the test gene, $\boldsymbol{w}$ denotes cis-eQTL effect sizes generated from $N\left(0, \sigma_{w}^{2} I\right)$ with variance $\sigma_{w}^{2}$ chosen to ensure a target gene expression heritability $\left(h_{e}^{2}\right)$, and $\boldsymbol{\varepsilon}_{\boldsymbol{E}}$ is the error term generated from $N\left(0,\left(1-h_{e}^{2}\right) \boldsymbol{I}\right)$.

Phenotype data are generated based on two models to mimic two different genetic architectures of complex traits that may be encountered with human data:

Model I: The genetic effects on the phenotype of interest are completely driven by genetically regulated gene expression (GReX), where SNP effect sizes are of a linear function of their corresponding cis-eQTL effect sizes as assumed by Burden-TWAS methods $[8,10,11]$. Phenotype data are generated from the following equation

$$
\boldsymbol{Y}=\phi \boldsymbol{E}_{g}+\boldsymbol{\varepsilon}_{Y}=r\left(\boldsymbol{G} \boldsymbol{w}+\boldsymbol{\varepsilon}_{\boldsymbol{E}}\right)+\boldsymbol{\varepsilon}_{Y}, \boldsymbol{\varepsilon}_{Y} \sim N\left(0,\left(1-h_{p}^{2}\right) \boldsymbol{I}\right),
$$

where $\boldsymbol{E}_{\boldsymbol{g}}$ is the gene expression generated from Eq (7) and $r=\sqrt{h_{p}^{2} / \operatorname{Var}\left(\boldsymbol{E}_{g}\right)}$ is a scalar chosen to ensure a target phenotype variation proportion due to gene expression $\left(h_{p}^{2}\right)$.

Model II: The magnitudes of SNP effect sizes on the phenotype are no longer a linear function of corresponding cis-eQTL effect sizes as in Model I, but rather are derived randomly from a distribution whose variance is a linear function of the squares of such effects. By doing 
this, the SNP effect sizes on phenotype are a function of cis-eQTL effect sizes but the assumption of a linear relationship between the two (assumed by Burden-TWAS) is relaxed. Phenotype data are generated from the following equation

$$
\boldsymbol{Y}=\boldsymbol{G} \boldsymbol{\beta}+\boldsymbol{\varepsilon}_{\boldsymbol{Y}}, \boldsymbol{\varepsilon}_{Y} \sim N\left(0,\left(1-h_{p}^{2}\right) \boldsymbol{I}\right),
$$

where $\boldsymbol{G}$ denotes the genotype matrix of randomly selected true causal SNPs that are also true causal cis-eQTL as in Eq (7), respective SNP effect sizes are generated from $\beta_{i} \sim N\left(0, r w_{i}^{2}\right)$ with corresponding cis-eQTL effect size $w_{i}$ as used in Eq (7) and $r$ chosen to ensure a phenotype heritability $h_{p}^{2}$.

We considered scenarios with various proportions of causal cis-eQTL/SNPs $p_{\text {causal }}=(0.001$, $0.01,0.1,0.2)$ for the test gene, and various combinations of expression heritability $\left(h_{e}^{2}\right)$ and phenotype variance proportion/heritability $\left(h_{p}^{2}\right)$ that were chosen to ensure TWAS power fall-

ing within the range of $(25 \%, 90 \%)$. The values of $p_{\text {causal }}$ and $h_{p}^{2}$ were taken as $\left(p_{\text {causal }}, h_{p}^{2}\right)=$ $((0.001,0.2),(0.01,0.3),(0.1,0.4),(0.2,0.5))$ for simulating phenotypes from Model I, while taken as $\left(p_{\text {causal }}, h_{p}^{2}\right)=((0.001,0.1),(0.01,0.1),(0.1,0.15),(0.2,0.15))$ for simulating phenotypes from Model II.

We used 499 ROS/MAP samples as training data that were also used as training data in our application studies, and randomly selected 1,232 ROS/MAP samples as test data. To show the power performance with respect to test sample size, we considered different test sample sizes $(400,800,1232)$ under the scenario with $p_{\text {causal }}=0.2$.

We estimated cis-eQTL effect sizes from training data by using PrediXcan and TIGAR DPR methods and then conducted Burden-TWAS and VC-TWAS with individual-level and summary-level test GWAS data. We note that Burden-TWAS using PrediXcan weights is equivalent to the PrediXcan method [10] while Burden-TWAS using DPR weights is equivalent to the TIGAR method [8]. We also compared Burden-TWAS and VC-TWAS with the CoMM method under simulation settings with $p_{\text {causal }}=0.2$ (we did not consider additional simulation settings because of the computational demands of CoMM).

For each scenario, we repeated simulations for 1,000 times and obtained the power as the proportion of simulations that had test $\mathrm{p}$-value $<2.5 \times 10^{-6}$ (genome-wide significance threshold for gene-based test). Additionally, we simulated phenotype under the null hypothesis $Y \sim N$ $(0,1)$ for $10^{6}$ times and evaluated type I errors of Burden-TWAS and VC-TWAS, using variant weights derived from PrediXcan and DPR methods.

For each scenario with VC-TWAS, we considered both the original form of the test as well as the alternate computationally-efficient form that considered only the filtered set of variants with cis-eQTL effect size estimates greater than the median effect size value in each simulation (about 50\% SNPs).

\section{Results}

\section{Simulation results}

We compared the performance of VC-TWAS and Burden-TWAS using PrediXcan weights (ciseQTL effect size estimates by Elastic-Net penalized regression) and DPR weights (cis-eQTL effect size estimates by DPR) under various scenarios. We also evaluated the performance of VC-TWAS and Burden-TWAS using filtered DPR weights as described in Methods. Under the scenario with $p_{\text {causal }}=0.2$, we considered different test sample sizes $(400,800,1232)$, and compared VC-TWAS with CoMM using both individual-level and summary level test data with 1232 test samples.

First, we compared TWAS power for studying phenotypes simulated from Model I that assumed SNP effect sizes on phenotypes were of a linear function of their corresponding cis- 
eQTL effect sizes. When $p_{\text {causal }}=(0.001,0.01)$ with sparse true causal signals (S1A Fig),

VC-TWAS had comparable power with Burden-TWAS and both TWAS methods using PrediXcan weights achieved higher power compared to using DPR weights. When $p_{\text {causal }}>0.01$, we observed that both TWAS methods using DPR weights achieved higher power compared to using PrediXcan weights. These results are consistent with the previous TIGAR paper [8]. This is because DPR method is preferred for modeling quantitative gene expression traits when a gene harbors a considerable proportion of true cis-eQTL with relatively smaller effect sizes, e.g., scenarios with $\mathrm{p}_{\text {causal }}>0.01$ in our simulation studies. As expected, with $\mathrm{p}_{\text {causal }}>0.01$ (S1A Fig, Fig 1A), Burden-TWAS methods outperformed VC-TWAS under Model I which meets the assumptions by Burden-TWAS methods. Across all considered scenarios, all TWAS methods using filtered DPR weights had similar performance as using complete DPR.

A) Model I

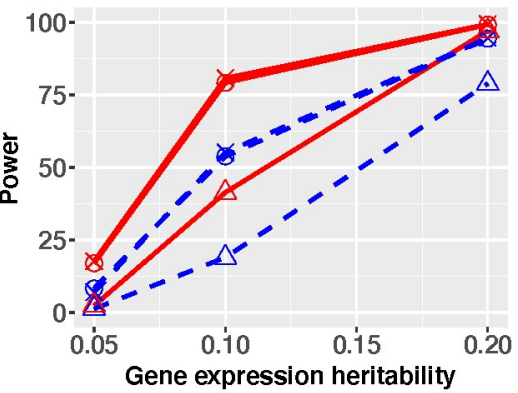

C) Model I

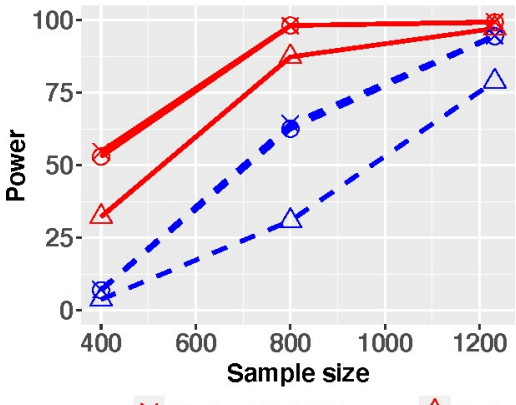

* Burden-TWAS DPR $\ominus$ Burden-TWAS_filtered_DPR $\nsucc$ vc-TWAS_DPR

E) Model I

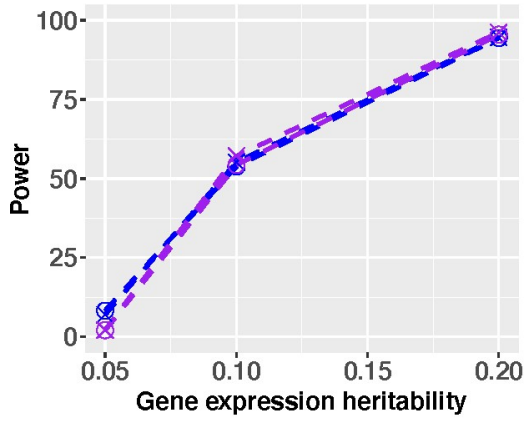

B) Model II

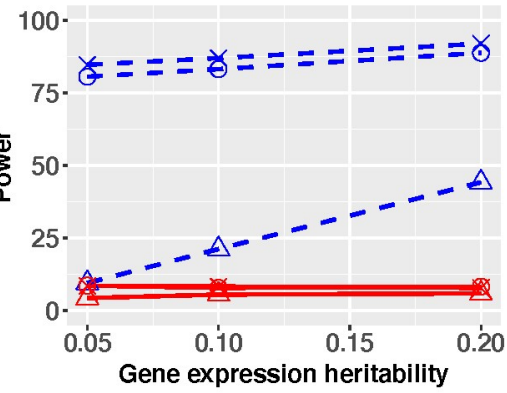

D) Model II

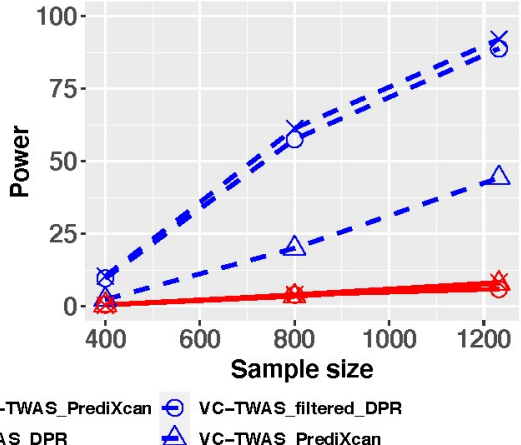

F) Model II

100

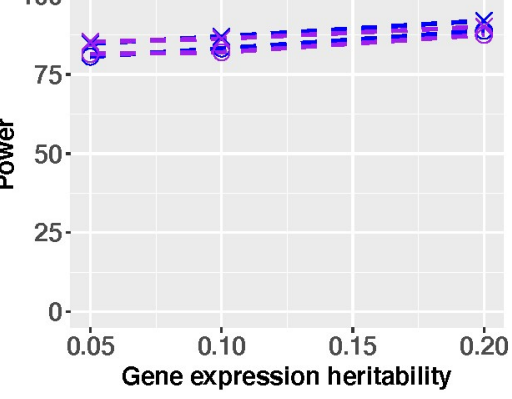

$\star$ VG-TWAS_DPR $\ominus$ vG-TWAS_DPR_f $\approx$ VG-TWAS-SS $\ominus$ vc-TWAS-SS_ $f$

Fig 1. Power comparison of Burden-TWAS and VC-TWAS methods under simulation scenarios with $20 \%$ true causal eQTL for gene expression (i.e., $\boldsymbol{p}_{\text {causal }}=\mathbf{0 . 2}$ ) in the test gene. Phenotypes simulated from Model I (A, C, E) and Model II (B, D, F) were considered. Various gene expression heritability and various types of SNP weights were considered, including those derived from PrediXcan method, DPR method, and filtered DPR weights in panel (A, B). Various test sample sizes $(400,800,1232)$ were considered in panel $(B, D)$. The VC-TWAS approach of using only summary-level GWAS data were validated in panel (E, F).

https://doi.org/10.1371/journal.pgen.1009482.g001 
Second, we compared TWAS power for studying phenotypes simulated from Model II that assumes variances of SNP effect sizes on phenotype were of a linear function of the squared values of their corresponding cis-eQTL effect sizes. As shown in Figs 1B and S1B, we found that the VC-TWAS obtained higher power than Burden-TWAS across all scenarios. Especially, with $p_{\text {causal }} \geq 0.01$, the power by VC-TWAS using DPR weights was double of that using PrediXcan weights on average ( $88.99 \%$ vs. $38.45 \%$ ), while the power of Burden-TWAS using DPR weights was comparable with using PrediXcan weights except when $\mathrm{p}_{\text {causal }}=0.001$. When $p_{\text {cau- }}$ $s a l=0.001$ and $h_{e}^{2} \in(0.1,0.2)$ (S1B Fig), both TWAS approaches using PrediXcan weights performed better than using DPR weights. Again, across all considered scenarios, TWAS using filtered DPR weights had similar performance to that using complete DPR.

Third, we compared VC-TWAS and Burden-TWAS with different test sample sizes (400, $800,1232)$ under the scenarios with $\left(p_{\text {causal }}=0.2, h_{e}^{2}=0.2\right)$. As shown in Figs $1 \mathrm{C}$ and $1 \mathrm{D}$, the power of VC-TWAS and Burden-TWAS increased with respect to the test sample size in both simulation models. Interestingly, for model II, the magnitude of the power difference between VC-TWAS and Burden-TWAS became more pronounced with increasing sample size.

Fourth, to validate the VC-TWAS approach for using only summary-level GWAS data, we compared VC-TWAS with DPR weights by using individual-level and summary-level GWAS data, under the scenarios with $p_{\text {causal }}=0.2$. As shown in Figs $1 \mathrm{E}$ and $1 \mathrm{~F}, \mathrm{VC}$-TWAS using summary-level GWAS data (VC-TWAS-SS) performed equivalent as using individual-level data under both simulation models. Additionally, VC-TWAS using filtered DPR weights and summary-level GWAS data (VC-TWAS_f) still had similar performance to that using complete DPR weights.

Fifth, we also compared VC-TWAS with CoMM $[20,21]$ using individual-level and CoMM-SS using summary-level GWAS data under the scenarios with $p_{\text {causal }}=0.2$ (S2 Fig). Although CoMM and CoMM-SS outperformed VC-TWAS, CoMM costed up to 600x more computation time and CoMM-SS costed up to 250x more computation time than VC-TWAS for testing a gene with $\sim 5 \mathrm{~K}$ test SNPs (S1 Table). Take typical genes with $\sim 2 \mathrm{~K}-5 \mathrm{~K}$ test SNPs as examples, VC-TWAS costed $\sim 20$ s versus $\sim 38,037$ s by CoMM using individual-level GWAS data, and VC-TWAS costed $\sim 3 \mathrm{~s}$ versus $\sim 373$ s by CoMM-SS using summary-level GWAS data, by a single-thread computation with 4 cores (32GB memory) on a 2.10GHZ CPU (16-node Intel Xeon node). As the number of the SNPs in a gene increases, the difference in computation run times between VC-TWAS and CoMM/CoMM-SS become even more pronounced.

Last but not least, to evaluate type I error of Burden-TWAS and VC-TWAS, we conducted $10^{6}$ times simulations under the null hypothesis where phenotypes were not associated with genetic data of the test gene. Without loss of generality, we used gene expression data simulated with $p_{\text {causal }}=0.2, h_{e}^{2}=0.1$ and generated phenotypes randomly from a $N(0,1)$ distribution. We evaluated type I errors (Table 1) with multiple significant levels $\left(10^{-2}, 10^{-4}\right.$, $\left.2.5 \times 10^{-6}\right)$, demonstrating that both TWAS approaches had type I errors well controlled across all considered significance levels. We also presented the quantile-quantile (QQ) plots of $\mathrm{p}$-values by all methods in S4 Fig.

Table 1. Type I errors under null simulation studies for Burden-TWAS and VC-TWAS with $p_{\text {causal }}=0.2, h_{e}^{2}=0.1$, using variant weights given by DPR, filtered DPR, and PrediXcan.

\begin{tabular}{l|l|l|l|l|l|l}
\hline \multirow{2}{*}{ Significance Level } & \multicolumn{4}{l}{ Burden-TWAS } & \multicolumn{3}{l}{ VC-TWAS } \\
\cline { 2 - 6 } & DPR & Filtered DPR & PrediXcan & DPR & Filtered DPR & PrediXcan \\
\hline $1.00 \times 10^{-2}$ & $9.82 \times 10^{-3}$ & $9.86 \times 10^{-3}$ & $9.27 \times 10^{-3}$ & $9.43 \times 10^{-3}$ & $9.46 \times 10^{-3}$ \\
\hline $1.00 \times 10^{-4}$ & $8.64 \times 10^{-5}$ & $8.44 \times 10^{-5}$ & $9.95 \times 10^{-5}$ & $8.64 \times 10^{-5}$ & $9.05 \times 10^{-5}$ \\
\hline $2.50 \times 10^{-6}$ & $2.00 \times 10^{-6}$ & $2.00 \times 10^{-6}$ & $2.00 \times 10^{-6}$ & $2.00 \times 10^{-6}$ & $1.00 \times 10^{-6}$ & $8.24 \times 10^{-5}$ \\
\hline
\end{tabular}

https://doi.org/10.1371/journal.pgen.1009482.t001 
To summarize, VC-TWAS performed similarly to Burden-TWAS for studying phenotypes simulated from Model I with sparse true causal eQTL, but outperformed Burden-TWAS for studying phenotypes simulated from model II. This is because the genetic architecture assumed under Model I is the one assumed by Burden-TWAS with linear relationship between SNP effect sizes on phenotype and cis-eQTL effect sizes. Whereas, Model II assumes a genetic architecture that is a function of cis-eQTL effect sizes but not explicitly a linear relationship (leading to more general models of effect). Generally, TWAS methods using DPR weights achieved higher power than using PrediXcan weights when $p_{\text {causal }} \geq 0.01$, which is consistent with previous studies [8]. Additionally, VC-TWAS using filtered DPR weights achieved similar power as using complete DPR weights, while saving up to $80 \%$ of computation time.

\section{Application studies of AD with individual-level GWAS data}

We applied VC-TWAS to the individual-level GWAS data of ROS/MAP and Mayo Clinic LOAD cohorts, using SNP weights (i.e., cis-eQTL effect sizes) generated by PrediXcan and filtered DPR methods with 499 ROS/MAP training samples that had both transcriptomic and genetic data profiled [8]. As suggested by previous studies [10,41], our TWAS results included genes with 5 -fold cross validation $(\mathrm{CV}) R^{2}>0.005$ for predicting quantitative gene expression traits by either PrediXcan or DPR. We obtained VC-TWAS p-values for 5,710 genes using PrediXcan weights and 12,650 genes using filtered DPR weights. Here, we roughly chose the threshold $10^{-4}$ to filter DPR weights in our VC-TWAS such that on average the variance component test considered $\sim 50 \%$ SNPs from the test gene region. Specifically, the median number of SNPs considered by VC-TWAS per gene is 2,872 for using filtered DPR weights and 6,632 for using complete DPR weights (S3 Fig).

Leveraging the clinical and postmortem $\mathrm{AD}$ data available in ROS/MAP, we were able to apply VC-TWAS to four clinical and pathologic AD phenotypes (S1D Text). We examined final cognitive status diagnosis of 1,436 decedents (AD Dementia $(\mathrm{N}=609)$ versus No Dementia $(\mathrm{N}=827)$ ), as well as three postmortem $\mathrm{AD}$ phenotypes including: continuous outcomes of $\beta$-amyloid load $(\mathrm{N}=1,294)$, PHFtau tangle density (tangles, $\mathrm{N}=1,303)$, and a global AD pathology $(\mathrm{N}=1,329)$. In the VC-TWAS of all four $\mathrm{AD}$ phenotypes, we adjusted for covariates of age, smoking status, sex, study group (ROS or MAP), education, and the top three principal components of ancestry.

With Mayo Clinic cohort, we conducted VC-TWAS for AD clinical diagnosis with 844 cases diagnosed with LOAD and 1,255 controls showed no signal of dementia, while adjusting for covariates age, sex, and top three principal components of ancestry. Since only the phenotype of $\mathrm{AD}$ clinical diagnosis was profiled by both ROS/MAP and Mayo Clinic cohorts (under different diagnosis criteria) and different sets of covariates were adjusted in VC-TWAS, we conducted meta-analysis with VC-TWAS summary statistics for each study by using Fisher's method (meta VC-TWAS) [42] to increase power with a larger sample size.

By meta VC-TWAS, we detected 13 significant risk genes with FDR $<0.05$ that were located within $\sim 2 \mathrm{MB}$ region around the well-known $\mathrm{AD}$ risk gene $A P O E$ on chromosome 19 (Fig 2A; Table 2). Seven of those significant genes were known risk genes of $\mathrm{AD}$ by previous GWAS (CLASRP, TOMM40, MARK4, CLPTM1, CEACAM19, RELB) [43,44] and BurdenTWAS using DPR weights (TRAPPC6A) [8].

As clinical $\mathrm{AD}$ diagnosis can be associated with $\mathrm{AD}$ related pathologies, in further analyses we investigated whether the genes found to be associated with clinical AD diagnosis (Table 2) were also associated with pathologic AD phenotypes. We examined the VC-TWAS p-values of these significant genes with respect to $\mathrm{AD}$ pathology phenotypes ( $\beta$-amyloid, tangles and global AD pathology) (S2 Table; Fig 2B; S5 and S6 Figs). Interestingly, 5 out of these 13 genes had at least one VC-TWAS p-value $<0.0013$ (Bonferroni correction with respect to 13 genes 
A)

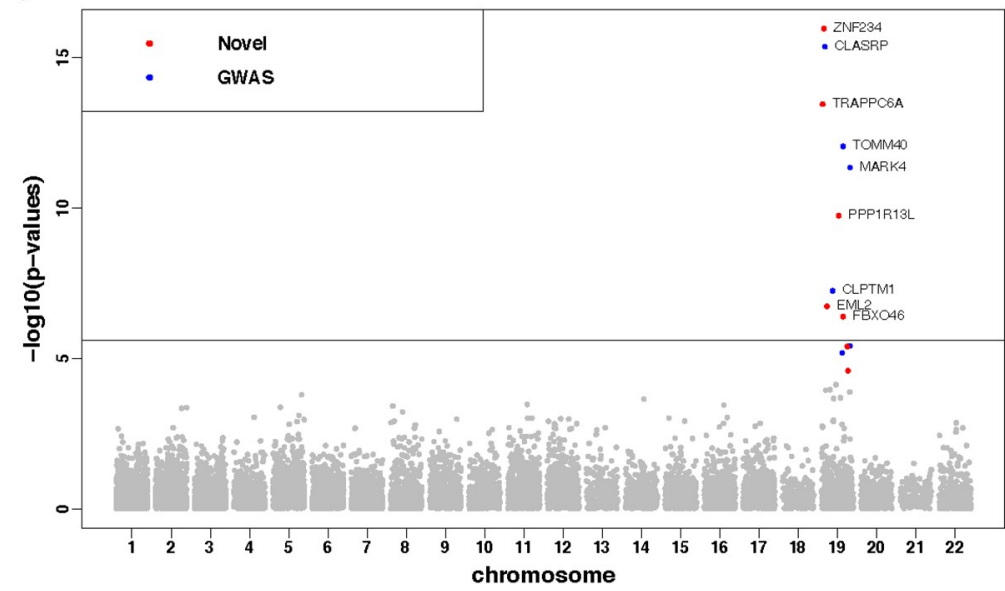

B)

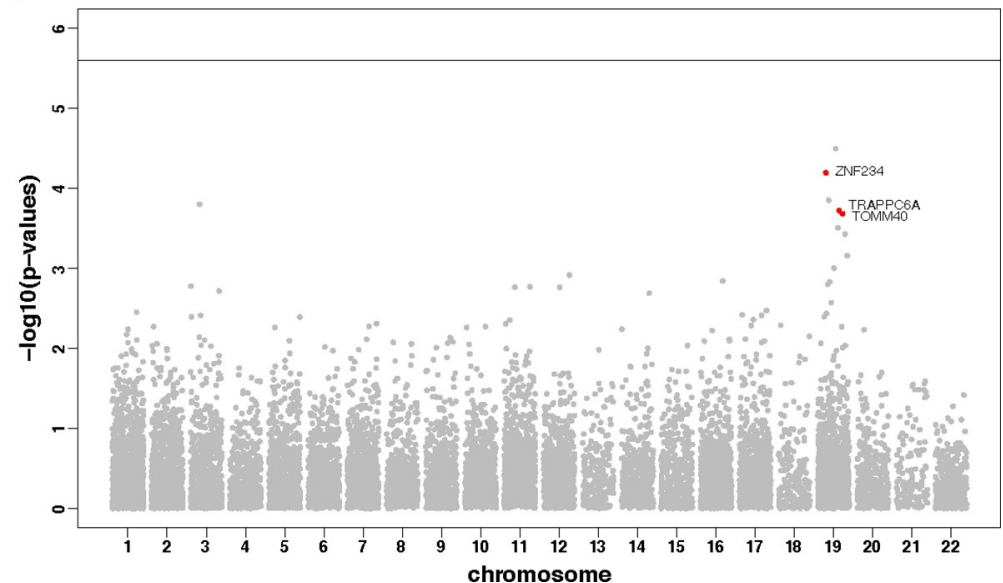

Fig 2. Manhattan plots of meta VC-TWAS for AD clinical diagnosis (A) and VC-TWAS of global AD pathology (B) with filtered DPR weights. Genes with FDR $<0.05$ are colored in (A), with red for novel risk genes and blue for known AD risk genes. Genes with FDR $<0.05$ in meta VC-TWAS of AD clinical diagnosis and $\mathrm{p}$-value $<0.0013$ in VC-TWAS of global AD pathology are colored in red in (B).

https://doi.org/10.1371/journal.pgen.1009482.g002

and $3 \mathrm{AD}$ pathology phenotypes) for one of the three $\mathrm{AD}$ pathology phenotypes. Particularly, genes ZNF234 and CLASRP have VC-TWAS p-value $<0.0013$ for all three AD pathology phenotypes, which are likely to be involved in the biological mechanisms of both $\beta$-amyloid and tangles. The other three genes (TRAPPC6A, TOMM40, CEACAM19) have VC-TWAS p-value $<0.0013$ for $\beta$-amyloid and global AD pathology, which are likely to be involved only in the biological mechanism of $\beta$-amyloid.

For example, the top significant gene ZNF234 (with FDR $=1.40 \times 10^{-12}$ ) by meta VC-TWAS of $\mathrm{AD}$ clinical diagnosis is also the top significant gene ( $\mathrm{p}$-value $=2.10 \times 10^{-4}$ ) by VC-TWAS of $\beta$-amyloid, the second most significant gene $\left(\mathrm{p}\right.$-value $\left.=6.39 \times 10^{-5}\right)$ by VC-TWAS of global AD pathology, and has $\mathrm{p}$-value $=1.06 \times 10^{-3}$ by VC-TWAS of tangles. These results showed that the genetic factor of gene ZNF234 on AD could be potentially mediated through its gene expression, and the expression of this gene could be also potentially involved in the mechanisms of both $\mathrm{AD}$ pathology indices of $\beta$-amyloid and tangles. Besides $\mathrm{AD}$, gene ZNF234 is also a known risk gene for lipid traits [45]. The genetically regulated gene expression of this gene might also affect lipid traits, thus leading to a pleiotropy phenomenon of AD and lipid traits [46]. Additionally, ZNF234 is known to be involved in the super pathway of gene expression 
Table 2. Significant genes for phenotype AD clinical diagnosis by meta VC-TWAS with filtered DPR weights. Significant genes have FDR $<0.05$ by meta-TWAS with ROS/MAP and Mayo Clinic cohorts. AD risk genes identified by previous GWAS are shaded in grey.

\begin{tabular}{l|l|l|l|l|l}
\hline Gene name & CHR & Start & End & P-value & FDR \\
\hline ZNF234 $^{a}$ & 19 & $44,645,710$ & $44,664,462$ & $1.11 \times 10^{-16}$ & $1.40 \times 10^{-12}$ \\
\hline CLASRP $^{a}$ & 19 & $45,542,298$ & $45,574,214$ & $4.44 \times 10^{-16}$ & $2.81 \times 10^{-12}$ \\
\hline TRAPPC6A $^{a}$ & 19 & $45,666,187$ & $45,681,485$ & $3.60 \times 10^{-14}$ & $1.52 \times 10^{-10}$ \\
\hline TOMM40 $^{a}$ & 19 & $45,394,477$ & $45,406,935$ & $9.05 \times 10^{-13}$ & $2.86 \times 10^{-9}$ \\
\hline MARK4 & 19 & $45,754,550$ & $45,808,541$ & $4.62 \times 10^{-16}$ & $1.17 \times 10^{-8}$ \\
\hline PPP1R13L & 19 & $45,882,892$ & $45,909,607$ & $1.82 \times 10^{-10}$ & $3.84 \times 10^{-7}$ \\
\hline CLPTM1 & 19 & $45,457,848$ & $45,496,598$ & $5.71 \times 10^{-8}$ & $1.03 \times 10^{-4}$ \\
\hline EML2 & 19 & $46,112,660$ & $46,148,726$ & $1.88 \times 10^{-7}$ & $2.97 \times 10^{-4}$ \\
\hline FBXO46 & 19 & $46,213,887$ & $46,234,151$ & $4.13 \times 10^{-7}$ & $5.80 \times 10^{-4}$ \\
\hline GEACAMR $19^{a}$ & 19 & $45,174,724$ & $45,187,631$ & $3.93 \times 10^{-6}$ & $4.68 \times 10^{-3}$ \\
\hline RELB & 19 & $46,171,502$ & $46,185,704$ & $4.07 \times 10^{-6}$ & $4.68 \times 10^{-3}$ \\
ZNF225 & 19 & $45,504,695$ & $45,541,452$ & $6.63 \times 10^{-6}$ & $6.99 \times 10^{-3}$ \\
\hline
\end{tabular}

a: Genes with significant p-values $<0.0013$ (Bonferroni correction with respect to 13 genes and 3 phenotypes) for at least one AD pathology phenotype

and is annotated with the Gene Ontology term of nucleic acid binding and DNA-binding transcription factor activity [47].

Another significant gene of interest is TOMM40, which has FDR $=2.86 \times 10^{-9}$ by meta VC-TWAS for AD dementia and VC-TWAS p-values $=\left(4.44 \times 10^{-4}, 6.95 \times 10^{-2}, 1.91 \times 10^{-4}\right)$ for $\beta$-amyloid, tangles, and global AD pathology, respectively. These findings suggest that the association of this well-known AD risk gene TOMM40 [48] could be mediated through its gene expression via $\beta$-amyloid but not tangles.

For all SNPs considered by meta VC-TWAS of genes ZNF234 and TOMM40 for studying AD clinical diagnosis, we colocalized the meta GWAS results for $\mathrm{AD}$ clinical diagnosis with ROS/MAP and Mayo Clinic cohorts and the corresponding DPR weight (i.e., cis-eQTL effect size) magnitude. Interestingly, we found that the VC-TWAS association of these two genes were likely to be driven by SNPs around APOE/TOMM40 loci that also possessed major ciseQTL effect size magnitudes (S7 Fig).

In addition, our VC-TWAS identified a significant gene HSPBAP1 $(\mathrm{FDR}=0.058)$ for tangles (S5B Fig). As shown by previous studies, mRNA of gene HSPBAP1 was abnormally expressed in the anterior temporal neocortex of patients with intractable epilepsy [49]. Based on our VC-TWAS results, gene HSPBAP1 might not have a significant genetic effect on AD dementia, but might have a significant effect on tangle pathology ( $\mathrm{p}$-value $=4.57 \times 10^{-6}$ ) and may account for the increasing recognition of non-cognitive $\mathrm{AD}$ phenotypes [50]. This provides support about that gene HSPBAP1 could be involved in the mechanism of brain pathology tangles and other neurological diseases such as intractable epilepsy [49,51].

We also applied VC-TWAS with PrediXcan weights, but no significant genes were identified with FDR $<0.05$ (S8, S9 and S10 Figs), neither the genes with smallest p-values were proximal to APOE. In contrast, our results by VC-TWAS with filtered DPR weights provided potential biological interpretations for several known $\mathrm{AD}$ risk genes via gene expression and for their associations with both clinical and pathologic $\mathrm{AD}$ phenotype.

\section{Application studies of AD with summary-level GWAS data}

Next, we applied VC-TWAS to the stage1 summary-level GWAS data of AD from IGAP [3], which has a much larger sample size ( $~ 54 \mathrm{~K}$ with $17,008 \mathrm{AD}$ cases and 37,154 controls). We 
considered filtered cis-eQTL DPR weights as used in the above applications with individuallevel GWAS data, as well as the SNP weights of both cis- and trans- eQTL generated by the BGW-TWAS method [30]. LD covariance matrices from ROS/MAP individual-level GWAS data were used for implementing VC-TWAS with summary-level GWAS data.

Using filtered cis-eQTL DPR weights. By using the filtered cis-eQTL DPR weights, we identified 57 significant risk genes with FDR $<0.05$ by VC-TWAS (Fig 3A; S3 Table and S11A Fig), including the most well-known $\mathrm{AD}$ risk genes TOMM40 and APOE, along with 45 genes
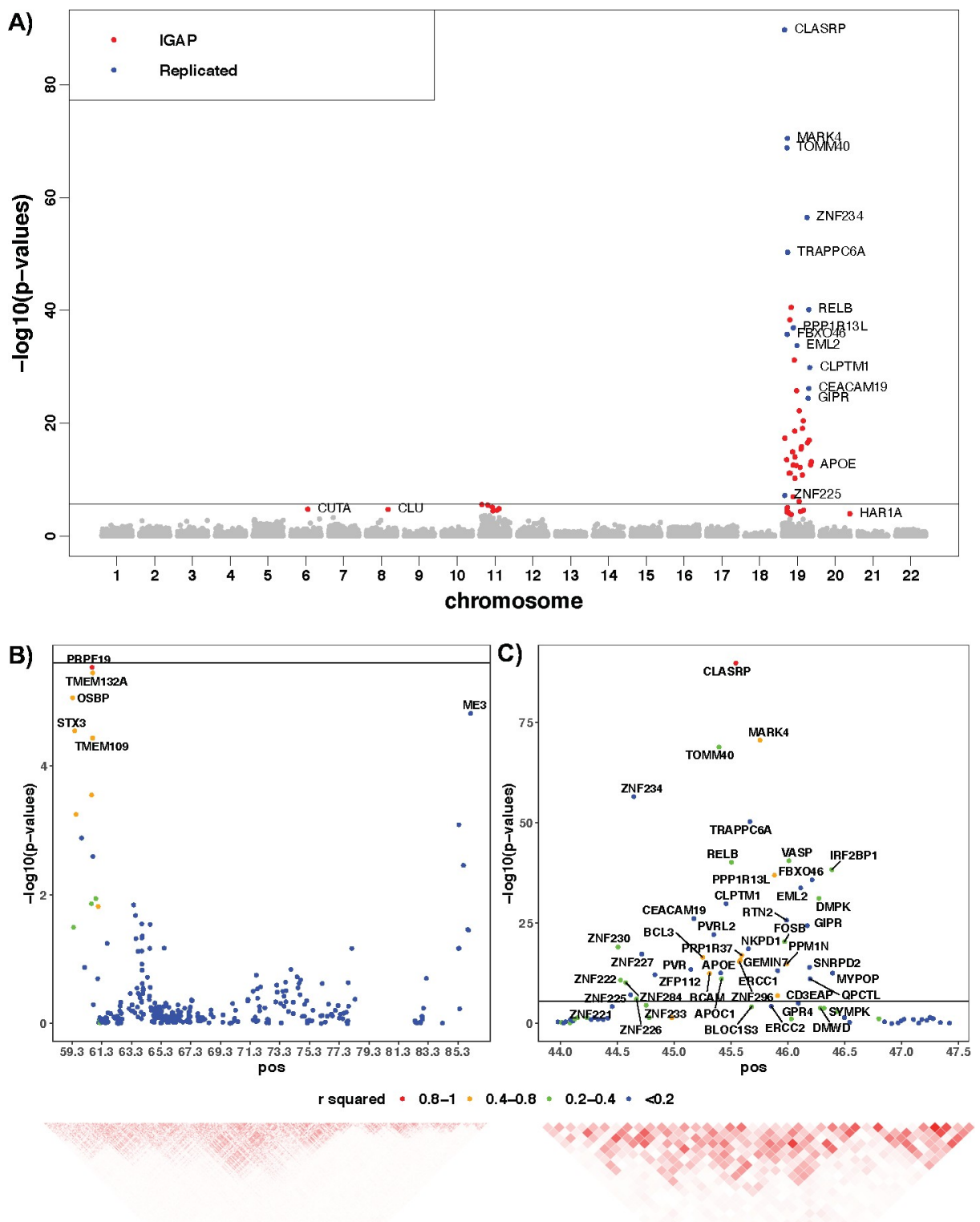

Fig 3. Manhattan plots of VC-TWAS using IGAP summary data with filtered cis-eQTL DPR weights (A) and TWAS locus zoom plots for the loci on chromosome 11 (B) and chromosome 19 (C). Significant genes with FDR $<0.05$ are colored, with red for significant ones only identified by using IGAP summary data and blue for the ones replicating our VC-TWAS findings using the individual-level GWAS data of ROS/MAP and Mayo Clinic cohort. Between gene $R^{2}$ in (B, C) were calculated with respect to GReX values. The $R^{2}$ in locus zoom plot denoted by various colors for the dots is the squared correlation of GReX between the most significant gene and other neighborhood genes. The heatmap is based on the squared correlation matrix of GReX.

https://doi.org/10.1371/journal.pgen.1009482.g003 
that were located within $\sim 2 \mathrm{MB}$ region around those two risk genes. Other significant genes are distributed on chromosome 6, 8, 11 and 20 (Fig 3A). Among these 57 significant genes, 20 genes were known risk genes by previous GWAS $[43,44]$ (S3 Table). Genes detected by VC-TWAS using the individual-level GWAS data of ROS/MAP and Mayo Clinic cohorts were all replicated (S3 Table). Burden-TWAS using IGAP summary data and filtered cis-eQTL DPR weights ( 33 Table) also identified 22 out of 57 of these significant genes.

Since most significant genes are nearby genes on chromosome 11 and 19, we made analogous TWAS locus zoom plots for these two major loci in Fig 3B and 3C, where the between gene $R^{2}$ were calculated with respect to the predicted GReX values using the individual-level GWAS data of ROS/MAP and Mayo Clinic cohorts. We observed that most significant genes do have highly correlated GReX values, showing that nearby significant TWAS associations are likely to be not independent. For the locus on chromosome 19 (Fig 3C), we can see that the $R^{2}$ between gene MARK4 (and a few other genes shown in orange color) and the top significant gene CLASRP is greater than 0.4 . The significant genes in blue colors are likely to be independent associations from the top significant gene CLASRP. For the locus on chromosome 11 (Fig 3B), significant genes TMEM132A, OSBP, STX3, and TMEM109 are highly correlated with the top significant gene $P R P F 19$, while gene $M E 3$ tend to be another independent association.

Using cis- and trans- eQTL weights generated by BGW-TWAS. To provide a complementary list of significant genes by considering both cis- and trans- eQTL, we conducted VC-TWAS with the IGAP summary data using the cis- and trans- eQTL weights generated by the BGW-TWAS method [30]. We detected total 71 significant genes with FDR $<0.05$ (Fig 4; S4 Table; S11B Fig), among which 6 genes were identified by GWAS [44,52-55] and 22 genes were shown to be related with $\mathrm{AD}$ or other neurological diseases by previous studies and (Table 3).

For example, gene ARHGEF2 on chromosome 1 (with FDR $=2.46 \times 10^{-13}$ ) is shown to be interact with all four members of the MARK family including MARK4 on chromosome 19 which has an emerging tole in the phosphorylation of MAPT/TAU in Alzheimer's disease and identified by VC-TWAS using cis-eQTL DPR weights [56]. Gene GAS5 (with FDR $=3.11 \times 10^{--}$ ${ }^{19}$ ) was shown to have a novel role in microglial polarization and the pathogenesis of demyelinating diseases which suggested the potential therapeutic benefit of targeting GAS5 for the

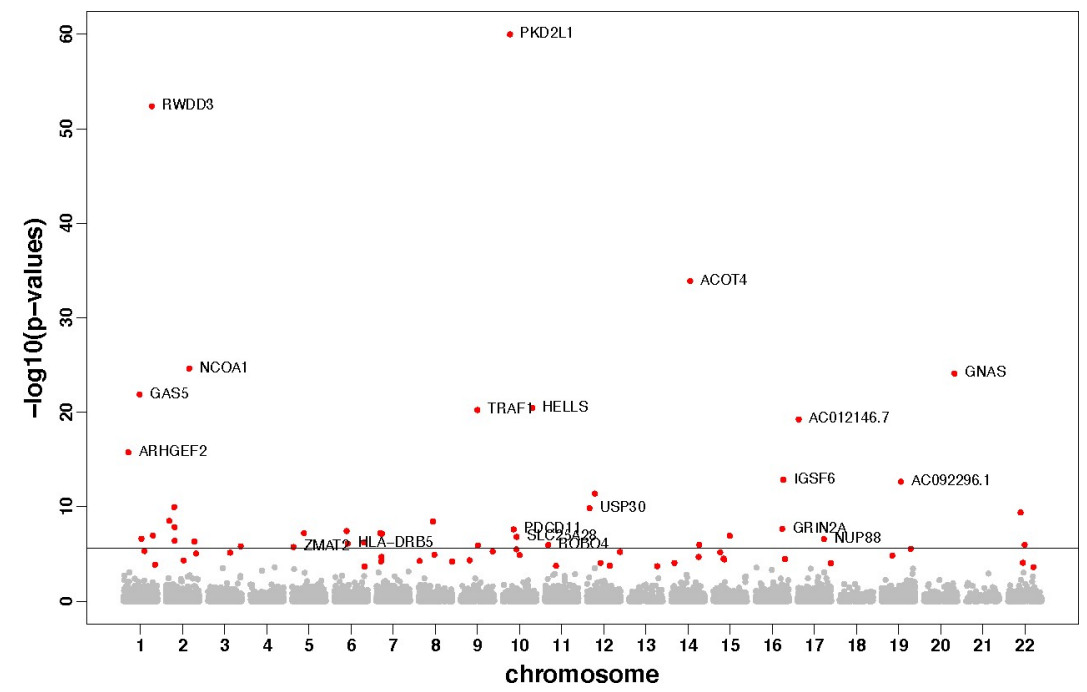

Fig 4. Manhattan plot of VC-TWAS using IGAP summary data with BGW cis- and trans- eQTL weights. Significant genes with FDR $<0.05$ are colored.

https://doi.org/10.1371/journal.pgen.1009482.g004 
Table 3. Significant genes identified by VC-TWAS using IGAP summary statistics data with BGW cis- and trans- eQTL weights, which are either known GWAS loci or shown to be related with AD or other neurological diseases by previous studies. AD risk genes identified by previous GWAS are shaded in grey.

\begin{tabular}{|c|c|c|c|c|c|}
\hline Gene Name & CHROM & Start & End & P-value & FDR \\
\hline$A R H G E F 2^{a}$ & 1 & $155,916,644$ & $155,966,129$ & $1.75 \times 10^{-16}$ & $2.46 \times 10^{-13}$ \\
\hline GAS5 $^{a}$ & 1 & $173,833,037$ & $173,838,020$ & $1.33 \times 10^{-22}$ & $3.11 \times 10^{-19}$ \\
\hline $\mathrm{NCOA}^{a}{ }^{a}$ & 2 & $24,714,782$ & $24,993,571$ & $2.42 \times 10^{-25}$ & $8.51 \times 10^{-22}$ \\
\hline CENPO $^{a}$ & 2 & $25,016,004$ & $25,045,245$ & $4.07 \times 10^{-7}$ & $1.85 \times 10^{-4}$ \\
\hline SLC4A10 ${ }^{a}$ & 2 & $162,280,842$ & $162,841,792$ & $8.80 \times 10^{-6}$ & $2.58 \times 10^{-3}$ \\
\hline MAN2A1 & 5 & $109,025,066$ & $109,205,326$ & $6.30 \times 10^{-8}$ & $3.70 \times 10^{-5}$ \\
\hline ZMAT2 $^{a}$ & 5 & $140,079,746$ & $140,086,266$ & $1.83 \times 10^{-6}$ & $6.43 \times 10^{-4}$ \\
\hline$H L A-D R B 5^{a}$ & 6 & $32,485,119$ & $32,498,064$ & $7.81 \times 10^{-7}$ & $3.23 \times 10^{-4}$ \\
\hline$A H R^{a}$ & 7 & $17,338,245$ & $17,385,776$ & $2.11 \times 10^{-5}$ & $5.62 \times 10^{-3}$ \\
\hline JAZF1 & 7 & $27,870,191$ & $28,220,362$ & $7.49 \times 10^{-8}$ & $4.22 \times 10^{-5}$ \\
\hline $\mathrm{ACHE}^{a}$ & 7 & $100,487,614$ & $100,494,594$ & $5.93 \times 10^{-5}$ & $1.39 \times 10^{-2}$ \\
\hline$D L G A P 2^{a}$ & 8 & $1,449,530$ & $1,656,642$ & $1.18 \times 10^{-5}$ & $3.38 \times 10^{-3}$ \\
\hline$R F X 3^{a}$ & 9 & $3,218,296$ & $3,526,004$ & $4.69 \times 10^{-5}$ & $1.16 \times 10^{-2}$ \\
\hline$S L C 25 A 28^{a}$ & 10 & $101,370,281$ & $101,380,535$ & $1.55 \times 10^{-7}$ & $7.79 \times 10^{-5}$ \\
\hline$P D C D 11^{a}$ & 10 & $105,156,404$ & $105,206,049$ & $2.52 \times 10^{-8}$ & $1.69 \times 10^{-5}$ \\
\hline $\mathrm{ROBO}^{a}$ & 11 & $124,753,586$ & $124,768,396$ & $1.13 \times 10^{-6}$ & $4.30 \times 10^{-4}$ \\
\hline$U_{S P} 30^{a}$ & 12 & $109,460,893$ & $109,525,831$ & $1.50 \times 10^{-10}$ & $1.41 \times 10^{-7}$ \\
\hline$P P P 1 R 3 E^{a}$ & 14 & $23,765,111$ & $23,772,057$ & $2.11 \times 10^{-5}$ & $5.62 \times 10^{-3}$ \\
\hline MTFMT $^{a}$ & 15 & $65,294,844$ & $65,321,977$ & $3.10 \times 10^{-5}$ & $8.09 \times 10^{-3}$ \\
\hline PARP6 & 15 & $72,533,521$ & $72,565,340$ & $6.77 \times 10^{-6}$ & $2.07 \times 10^{-3}$ \\
\hline GRIN2A ${ }^{a}$ & 16 & $9,852,375$ & $10,276,611$ & $2.22 \times 10^{-8}$ & $1.56 \times 10^{-5}$ \\
\hline SETD6 $^{a}$ & 16 & $58,549,382$ & $58,554,431$ & $3.44 \times 10^{-5}$ & $8.81 \times 10^{-3}$ \\
\hline NUP88 ${ }^{a}$ & 17 & $5,264,257$ & $5,323,480$ & $2.65 \times 10^{-7}$ & $1.24 \times 10^{-4}$ \\
\hline NOS $2^{a}$ & 17 & $26,083,791$ & $26,127,555$ & $9.55 \times 10^{-5}$ & $2.07 \times 10^{-2}$ \\
\hline CEACAM19 & 19 & $45,174,723$ & $45,187,631$ & $1.46 \times 10^{-5}$ & $4.03 \times 10^{-3}$ \\
\hline APOC1 $^{a}$ & 19 & $45,417,920$ & $45,422,606$ & $2.80 \times 10^{-6}$ & $9.63 \times 10^{-4}$ \\
\hline
\end{tabular}

a: Genes shown to be related with $\mathrm{AD}$ or other neurological diseases by previous studies

treatment of neurological disorders including AD [57]. Gene NCOA1 (with FDR $8.51 \times 10^{-22}$ ) was shown to have pivotal roles in memory and learning [58]. SNPs in Gene PDCD11 (with $\mathrm{FDR}=1.69 \times 10^{-5}$ ) have been showed to be significantly associated with AD [59]. Gene APOC1 was detected by several GWASs [43,44], even when conditioning on APOE gene [60]. The presence of gene $A C H E$ (with $\mathrm{FDR}=1.39 \times 10^{-2}$ ) is a common feature in AD brain [61]. We also detected genes NUP88 [62], ROBO4 [63], DLGAP2 [64], AHR [65], PPP1R3E [66], RFX3 [67] and NOS2 [68] that were shown to possess biological link with AD. Additional identified genes USP30 [69], GRIN2A [70], SLC25A28 [71], HLA-DRB5 [72], ZMAT2 [73], SLC4A10 [74], MTFMT [75] and SETD6 [76] were shown to be related with neurological diseases by previous studies.

Overall, we show that VC-TWAS using BGW weights provide a complementary list of risk genes to those previously identified by considering additional trans- eQTL information.

\section{Discussion}

In this paper, we propose a novel variance-component TWAS (VC-TWAS) method that leverages eQTL effect sizes from reference transcriptome but does not assume a linear relationship between SNP effect sizes on phenotypes and cis-eQTL effect sizes. VC-TWAS is applicable to 
both quantitative and dichotomous outcomes and can further handle both individual-level and summary-level GWAS data. By implementing this VC-TWAS with cis-eQTL effect sizes estimated by DPR method $[8,13]$, we created a powerful test statistic that had good performance in simulation studies and obtained biologically meaningful TWAS results for both clinical and pathologic AD phenotypes. In particular, with the individual-level GWAS data of $\sim 3.4 \mathrm{~K}$ samples, we detected 13 TWAS genes for AD dementia, including the well-known GWAS risk gene TOMM40 and previously identified TWAS gene TRAPPC6A [8]. Moreover, 6 out of these 13 genes were identified by previous GWAS [43]. The pleiotropy effects of 5 of these genes with respect to $\mathrm{AD}$ dementia and indices of $\mathrm{AD}$ pathology demonstrated the possible biological mechanisms linking $\mathrm{AD}$ risk genes via $\beta$-amyloid and tangles with $\mathrm{AD}$ dementia.

By applying VC-TWAS with summary-level GWAS data of AD with a much larger sample size, we not only validated our findings with the individual-level GWAS data but also detected additional novel risk genes. In particular, by applying VC-TWAS with both cis- and transeQTL effect sizes estimated by the BGW-TWAS method, we identified a list of significant AD risk genes that complement risk genes identified by considering only cis-eQTL information.

To help users conduct our VC-TWAS method conveniently and efficiently, we added this method into our previously developed tool--Transcriptome Integrated Genetic Association Resource (TIGAR) [8]. The user has the option of estimating cis-eQTL effect sizes by either the PrediXcan method (i.e., Elastic-Net) [12] or nonparametric Bayesian DPR method [13], or using eQTL weights generated by previous studies. The VC-TWAS function works for using individual-level GWAS data to study continuous or dichotomous phenotypes, as well as using summary-level GWAS data.

Since the variance component test statistic used by VC-TWAS involves calculating and performing an eigen-decomposition of a genotypic kernel matrix, efficient computation is required (even when filtering variants to include only those variants with relatively larger magnitude of eQTL estimates) for obtaining the corresponding p-values in practice. Our TIGAR tool implements multi-threaded computation to take advantage of high-performance cloud computing clusters and enable practical computation for testing genome-wide genes with respect to reference transcriptome data of multiple tissue types. A typical VC-TWAS with $\sim 20 \mathrm{~K}$ genes can be accomplished within $\sim 80$ hours by using a single thread with $1 \mathrm{CPU}$ core and 32 GB memory.

Of course, current TWAS methods including our VC-TWAS still have their limitations. Because of genetic and transcriptomic heterogeneities across different ethnicities, one may have difficulty in translating eQTL effect size estimates across cohorts with different ethnicities [77]. That being said, VC-TWAS is likely more robust to this phenomenon than BurdenTWAS given the former relaxes the assumption of the latter of a linear relationship between the SNP effect size and eQTL effect in the transcriptome. Nevertheless, reference panels with diverse ethnicities and multiple tissue types are certainly needed to enhance TWAS to study complex diseases across different ancestral groups. We should note though that both ROS/ MAP and Mayo Clinic cohorts that we considered in this work have similar (European) origins.

Compared to the alternative CoMM and PMR-Egger methods that jointly model both reference and test data, VC-TWAS might be less powerful when the eQTL effect sizes are homogeneous in both reference and test cohorts. However, CoMM and PMR-Egger methods are derived for quantitative phenotypes and implement likelihood ratio tests under a maximum likelihood reference framework that are computationally expensive when thousands of cisSNPs need to be tested per gene. Although one may apply CoMM and PMR-Egger methods to dichotomous phenotypes by taking cases as 1's and controls as 0's, this may lead to inflated type I errors when population stratification leads to violation of the constant-residual variance 
assumption. The recently published generalized linear mixed model association test (GMMAT) method paper has shown this by both simulation and real studies and suggested that a logistic mixed model would be more appropriate for analyzing dichotomous traits [78]. Thus, VC-TWAS would be preferred because of computational efficiency when imputed or whole genome sequencing genotype data are considered for the reference panel, or when dichotomous traits are analyzed. We provide a summary table for pros and cons of existing popular TWAS methods in S5 Table.

Nevertheless, our novel technique allows a more flexible framework to account for the unknown genetic architectures underlying the relationships between SNPs and the phenotype of interest with efficient computation. Using simulated and real AD-related data, we show our method VC-TWAS provides the public a useful tool for illustrating the genetic etiology of complex diseases by providing a list of risk genes whose effects on phenotypes might be mediated through transcriptomes.

\section{Web resources}

VC-TWAS, https://github.com/yanglab-emory/VC_TWAS

TIGAR, https://github.com/yanglab-emory/TIGAR

PrediXcan, https://github.com/hakyim/PrediXcan

RADC Research Resource Sharing Hub, http://www.radc.rush.edu/

ROS/MAP data, https://www.synapse.org/\#!Synapse:syn3219045

MayoClinicLOAD data, https://www.synapse.org/\#!Synapse:syn2910256

GWAS catalog, https://www.ebi.ac.uk/gwas/

BGW weights of brain frontal cortex tissue, https://www.synapse.org/\#!Synapse: syn22316792

\section{Supporting information}

S1 Text. Details about PrediXcan's and TIGAR's approach of estimating cis-eQTL effect sizes (A, B), VC-TWAS approach with summary-level GWAS data (C), and ROS/MAP data (D). (DOCX)

S1 Fig. TWAS power comparison for VC-TWAS and Burden-TWAS with phenotypes simulated from Model I (A) and Model II (B). Various types of SNP weights were considered, including those derived from PrediXcan method, DPR method, and filtered DPR weights. In Model I, the combinations of causal probability and phenotype heritability are $\left(p_{\text {causal }}, h_{p}^{2}\right)=((0.001,0.2),(0.01,0.3),(0.1,0.4),(0.2,0.5))$. In Model II, the combinations of causal probability and phenotype heritability are $\left(p_{\text {causal }}, h_{p}^{2}\right)=((0.001,0.1),(0.01,0.1),(0.1,0.15),(0.2,0.15))$. (PDF)

S2 Fig. TWAS power comparison for VC-TWAS and CoMM with phenotypes simulated from Model I (A) and Model II (B) using individual-level and summary-level data under the scenarios with $p_{\text {causal }}=0.2$. (PDF)

S3 Fig. Box plot of the number of test SNPs considered by VC-TWAS of all genome-wide genes in the application studies of AD, with complete DPR weights and filtered DPR weights derived from the ROS/MAP training data.

(PDF) 
S4 Fig. Q-Q plots for VC-TWAS and Burden-TWAS with DPR weights, filtered DPR weights, and PrediXcan weights under null hypothesis, where quantitative gene expression traits were generated with $p_{\text {causal }}=0.2$ and $h_{e}^{2}=0.1$.

(PDF)

S5 Fig. Manhattan plots of VC-TWAS results with filtered DPR weights for studying quantitative AD pathology of $\beta$-Amyloid (A) and tangles (B). Genes with FDA $<0.05$ by meta VC-TWAS for studying AD clinical diagnosis are colored in red in (A) and top significant gene for studying tangles phenotype with FDR $=0.058$ is colored in red in (B). (PDF)

S6 Fig. Q-Q plots of VC-TWAS results with filtered DPR weights for studying $\beta$-amyloid, tangles, and global AD pathology with ROS/MAP cohort, as well as meta VC-TWAS results with filtered DPR weights for studying AD clinical diagnosis with ROS/MAP and Mayo Clinic cohorts.

(PDF)

S7 Fig. Locus zoom plots of GWAS results and the magnitude (i.e., absolute value) of ciseQTL effect size estimates by DPR method for SNPs that were considered by VC-TWAS of genes TOMM40 (A, B) and ZNF2334 (C, D). Filtered test SNPs with the cis-eQTL effect size magnitude $>10^{-4}$ were plotted here. SNPs with GWAS p-value $<5 \times 10^{-8}$ were colored in red in $(B, D)$, top significant SNPs by GWAS in $(A, C)$ were shown as the blue triangle in $(B, D)$. $(\mathrm{PDF})$

S8 Fig. Manhattan plots of VC-TWAS results with PrediXcan weights for studying AD clinical diagnosis (A) and global AD pathology (B).

(PDF)

S9 Fig. Manhattan plots of VC-TWAS results with PrediXcan weights for studying quantitative $\mathrm{AD}$ pathology of $\beta$-Amyloid (A) and tangles (B).

S10 Fig. Q-Q plots of VC-TWAS results with PrediXcan weights for studying $\beta$-amyloid, tangles, and global AD pathology with ROS/MAO cohort, as well as meta VC-TWAS results with PrediXcan weights for studying AD clinical diagnosis with ROS/MAP and Mayo Clinic cohorts.

(PDF)

S11 Fig. Q-Q plots of VC-TWAS results with cis-eQTL DPR filtered weights and BGW weights on IGAP GWAS summary statistics.

S1 Table. Average computation time per gene (in the unit of second) by all methods with individual and summary-level GWAS data by a single thread with 4 cores (32GB memory) from a 2.10GHZ CPU 16-core Intel Xeon computation node, for example genes that have test SNP numbers in respective range. (DOCX)

S2 Table. Genes with VC-TWAS p-value $<0.0013$ with respect to at least one AD pathology phenotype and FDR $<\mathbf{0 . 0 5}$ by meta VC-TWAS of AD clinical diagnosis. AD risk genes identified by previous GWAS are shaded in grey.

(DOCX) 
S3 Table. Significant genes identified by VC-TWAS using IGAP summary statistics with filtered cis-eQTL DPR weights. Significant genes were identified with FDR $<0.05$. AD risk genes identified by previous GWAS are shaded in grey.

(DOCX)

S4 Table. Novel significant genes identified by VC-TWAS using summary statistics with BGW weights on IGAP summary statistics.

(DOCX)

S5 Table. Pros and cons of existing popular TWAS methods.

(DOCX)

\section{Acknowledgments}

ROS/MAP study data were provided by the Rush Alzheimer's Disease Center, Rush University Medical Center, Chicago, IL. The MCADGC led by Dr. Nilüfer Ertekin-Taner and Dr. Steven G. Younkin, Mayo Clinic, Jacksonville, FL uses samples from the Mayo Clinic Study of Aging, the Mayo Clinic Alzheimer's Disease Research Center, and the Mayo Clinic Brain Bank.

\section{Author Contributions}

Conceptualization: Michael P. Epstein, Jingjing Yang.

Data curation: Shizhen Tang, Aron S. Buchman, Philip L. De Jager, David A. Bennett.

Formal analysis: Shizhen Tang.

Funding acquisition: Jingjing Yang.

Methodology: Shizhen Tang, Michael P. Epstein, Jingjing Yang.

Software: Shizhen Tang.

Writing - original draft: Shizhen Tang.

Writing - review \& editing: Aron S. Buchman, David A. Bennett, Michael P. Epstein, Jingjing Yang.

\section{References}

1. McCarthy MI, Abecasis GR, Cardon LR, Goldstein DB, Little J, loannidis JP, et al. Genome-wide association studies for complex traits: consensus, uncertainty and challenges. Nature reviews Genetics. 2008; 9(5):356-69. Epub 2008/04/10. https://doi.org/10.1038/nrg2344 PMID: 18398418.

2. Kettunen J, Tukiainen T, Sarin AP, Ortega-Alonso A, Tikkanen E, Lyytikainen LP, et al. Genome-wide association study identifies multiple loci influencing human serum metabolite levels. Nat Genet. 2012; 44(3):269-76. Epub 2012/01/31. https://doi.org/10.1038/ng.1073 PMID: 22286219; PubMed Central PMCID: PMC3605033.

3. Lambert JC, Ibrahim-Verbaas CA, Harold D, Naj AC, Sims R, Bellenguez C, et al. Meta-analysis of 74,046 individuals identifies 11 new susceptibility loci for Alzheimer's disease. Nature genetics. 2013; 45(12):1452-8. Epub 2013/10/29. https://doi.org/10.1038/ng.2802 PMID: 24162737; PubMed Central PMCID: PMC3896259.

4. Cannon ME, Mohlke KL. Deciphering the Emerging Complexities of Molecular Mechanisms at GWAS Loci. Am J Hum Genet. 2018; 103(5):637-53. Epub 2018/11/06. https://doi.org/10.1016/j.ajhg.2018.10. 001 PMID: 30388398; PubMed Central PMCID: PMC6218604.

5. He X, Fuller CK, Song Y, Meng Q, Zhang B, Yang X, et al. Sherlock: detecting gene-disease associations by matching patterns of expression QTL and GWAS. American journal of human genetics. 2013; 92(5):667-80. Epub 2013/05/07. https://doi.org/10.1016/j.ajhg.2013.03.022 PMID: 23643380; PubMed Central PMCID: PMC3644637. 
6. Nicolae DL, Gamazon E, Zhang W, Duan S, Dolan ME, Cox NJ. Trait-associated SNPs are more likely to be eQTLs: annotation to enhance discovery from GWAShttps://doi.org/10.1371/journal.pgen. 1000888 PMID: 20369019 PLoS genetics. 2010; 6(4):e1000888. Epub 2010/04/07. PubMed Central PMCID: PMC2848547.

7. Gamazon ER, Huang RS, Cox NJ, Dolan ME. Chemotherapeutic drug susceptibility associated SNPs are enriched in expression quantitative trait loci. Proc Natl Acad Sci U S A. 2010; 107(20):9287-92. Epub 2010/05/06. https://doi.org/10.1073/pnas.1001827107 PMID: 20442332; PubMed Central PMCID: PMC2889115

8. Nagpal S, Meng X, Epstein MP, Tsoi LC, Patrick M, Gibson G, et al. TIGAR: An Improved Bayesian Tool for Transcriptomic Data Imputation Enhances Gene Mapping of Complex Traits. The American Journal of Human Genetics. 2019; 105(2):258-66. https://doi.org/10.1016/j.ajhg.2019.05.018 PMID: 31230719

9. Consortium GT, Laboratory DA, Coordinating Center -Analysis Working G, Statistical Methods groupsAnalysis Working G, Enhancing Gg, Fund NIHC, et al. Genetic effects on gene expression across human tissues. Nature. 2017; 550(7675):204-13. Epub 2017/10/13. https://doi.org/10.1038/ nature24277 PMID: 29022597; PubMed Central PMCID: PMC5776756.

10. Gamazon ER, Wheeler HE, Shah KP, Mozaffari SV, Aquino-Michaels K, Carroll RJ, et al. A genebased association method for mapping traits using reference transcriptome data. Nat Genet. 2015; 47 (9):1091-8. Epub 2015/08/11. https://doi.org/10.1038/ng.3367 PMID: 26258848; PubMed Central PMCID: PMC4552594.

11. Gusev A, Ko A, Shi H, Bhatia G, Chung W, Penninx BW, et al. Integrative approaches for large-scale transcriptome-wide association studies. Nature genetics. 2016; 48(3):245-52. Epub 2016/02/09. https://doi.org/10.1038/ng.3506 PMID: 26854917; PubMed Central PMCID: PMC4767558.

12. Zou H, Hastie T. Regularization and Variable Selection via the Elastic Net. Journal of the Royal Statistical Society Series B (Statistical Methodology). 2005; 67(2):301-20.

13. Zeng $P$, Zhou $X$. Non-parametric genetic prediction of complex traits with latent Dirichlet process regression models. Nat Commun. 2017; 8(1):456. Epub 2017/09/08. https://doi.org/10.1038/s41467017-00470-2 PMID: 28878256; PubMed Central PMCID: PMC5587666.

14. Li YI, Wong G, Humphrey J, Raj T. Prioritizing Parkinson's disease genes using population-scale transcriptomic data. Nat Commun. 2019; 10(1):994. Epub 2019/03/03. https://doi.org/10.1038/s41467-01908912-9 PMID: 30824768; PubMed Central PMCID: PMC6397174.

15. Keys KL, Mak ACY, White MJ, Eckalbar WL, Dahl AW, Mefford J, et al. On the cross-population generalizability of gene expression prediction models. bioRxiv. 2019:552042. https://doi.org/10.1101/552042

16. Wu MC, Lee S, Cai T, Li Y, Boehnke M, Lin X. Rare-variant association testing for sequencing data with the sequence kernel association test. American journal of human genetics. 2011; 89(1):82-93. Epub 2011/07/09. https://doi.org/10.1016/j.ajhg.2011.05.029 PMID: 21737059; PubMed Central PMCID: PMC3135811.

17. Kwee LC, Liu D, Lin X, Ghosh D, Epstein MP. A powerful and flexible multilocus association test for quantitative traits. Am J Hum Genet. 2008; 82(2):386-97. Epub 2008/02/07. https://doi.org/10.1016/j. ajhg.2007.10.010 PMID: 18252219; PubMed Central PMCID: PMC2664991.

18. Wu MC, Kraft P, Epstein MP, Taylor DM, Chanock SJ, Hunter DJ, et al. Powerful SNP-set analysis for case-control genome-wide association studies. Am J Hum Genet. 2010; 86(6):929-42. Epub 2010/06/ 22. https://doi.org/10.1016/j.ajhg.2010.05.002 PMID: 20560208; PubMed Central PMCID: РМС3032061.

19. Ionita-Laza I, Lee S, Makarov V, Buxbaum JD, Lin X. Sequence kernel association tests for the combined effect of rare and common variants. Am J Hum Genet. 2013; 92(6):841-53. Epub 2013/05/21. https://doi.org/10.1016/j.ajhg.2013.04.015 PMID: 23684009; PubMed Central PMCID: PMC3675243.

20. Yeung K-F, Yang Y, Yang C, Liu J. CoMM: A Collaborative Mixed Model That Integrates GWAS and eQTL Data Sets to Investigate the Genetic Architecture of Complex Traits. Bioinformatics and Biology Insights. 2019; 13:117793221988143. https://doi.org/10.1177/1177932219881435 PMID: 31662603

21. Yang $Y$, Shi $X$, Jiao $Y$, Huang J, Chen $M$, Zhou X, et al. CoMM-S2: a collaborative mixed model using summary statistics in transcriptome-wide association studies. Bioinformatics. 2019; 36(7):2009-16. https://doi.org/10.1093/bioinformatics/btz880 PMID: 31755899

22. Yuan Z, Zhu H, Zeng P, Yang S, Sun S, Yang C, et al. Testing and controlling for horizontal pleiotropy with probabilistic Mendelian randomization in transcriptome-wide association studies. Nat Commun. 2020; 11(1):3861. Epub 2020/08/02. https://doi.org/10.1038/s41467-020-17668-6 PMID: 32737316; PubMed Central PMCID: PMC7395774.

23. Liu L, Zeng P, Xue F, Yuan Z, Zhou X. Multi-trait transcriptome-wide association studies with probabilistic Mendelian randomization. American journal of human genetics. 2021; 108(2):240-56. Epub 2021/ 01/13. https://doi.org/10.1016/j.ajhg.2020.12.006 PMID: 33434493. 
24. Bennett DA, Schneider JA, Arvanitakis Z, Wilson RS. Overview and findings from the religious orders study. Curr Alzheimer Res. 2012; 9(6):628-45. Epub 2012/04/05. https://doi.org/10.2174/ 156720512801322573 PMID: 22471860; PubMed Central PMCID: PMC3409291.

25. Bennett DA, Schneider JA, Buchman AS, Barnes LL, Boyle PA, Wilson RS. Overview and findings from the rush Memory and Aging Project. Curr Alzheimer Res. 2012; 9(6):646-63. Epub 2012/04/05. https:// doi.org/10.2174/156720512801322663 PMID: 22471867; PubMed Central PMCID: PMC3439198.

26. $\mathrm{Ng} \mathrm{B}$, White CC, Klein HU, Sieberts SK, McCabe C, Patrick E, et al. An xQTL map integrates the genetic architecture of the human brain's transcriptome and epigenome. Nat Neurosci. 2017; 20(10):1418-26. Epub 2017/09/05. https://doi.org/10.1038/nn.4632 PMID: 28869584; PubMed Central PMCID: PMC5785926.

27. Bennett DA, Buchman AS, Boyle PA, Barnes LL, Wilson RS, Schneider JA. Religious Orders Study and Rush Memory and Aging Project. J Alzheimers Dis. 2018; 64(s1):S161-S89. Epub 2018/06/06. https:// doi.org/10.3233/JAD-179939 PMID: 29865057; PubMed Central PMCID: PMC6380522.

28. Carrasquillo MM, Zou F, Pankratz VS, Wilcox SL, Ma L, Walker LP, et al. Genetic variation in $\mathrm{PCDH} 11 \mathrm{X}$ is associated with susceptibility to late-onset Alzheimer's disease. Nature genetics. 2009; 41 (2):192-8. Epub 2009/01/13. https://doi.org/10.1038/ng.305 PMID: 19136949; PubMed Central PMCID: PMC2873177.

29. Zou F, Chai HS, Younkin CS, Allen M, Crook J, Pankratz VS, et al. Brain Expression Genome-Wide Association Study (eGWAS) Identifies Human Disease-Associated Variants. PLOS Genetics. 2012; 8 (6):e1002707. https://doi.org/10.1371/journal.pgen.1002707 PMID: 22685416

30. Luningham JM, Chen J, Tang S, De Jager PL, Bennett DA, Buchman AS, et al. Bayesian Genome-wide TWAS method to leverage both cis- and trans- eQTL information through summary statistics. bioRxiv. 2020:2020.03.05.979187. https://doi.org/10.1016/j.ajhg.2020.08.022 PMID: 32961112

31. Liu D, Lin X, Ghosh D. Semiparametric regression of multidimensional genetic pathway data: leastsquares kernel machines and linear mixed models. Biometrics. 2007; 63(4):1079-88. Epub 2007/12/ 15. https://doi.org/10.1111/j.1541-0420.2007.00799.x PMID: 18078480; PubMed Central PMCID: PMC2665800.

32. Liu D, Ghosh D, Lin X. Estimation and testing for the effect of a genetic pathway on a disease outcome using logistic kernel machine regression via logistic mixed models. BMC Bioinformatics. 2008; 9:292. Epub 2008/06/26. https://doi.org/10.1186/1471-2105-9-292 PMID: 18577223; PubMed Central PMCID: PMC2483287.

33. Moschopoulos PG, Canada WB. The distribution function of a linear combination of chi-squares. Computers \& Mathematics with Applications. 1984; 10(4):383-6. https://doi.org/10.1016/0898-1221(84) 90066-X.

34. Lee S, Teslovich TM, Boehnke M, Lin X. General framework for meta-analysis of rare variants in sequencing association studies. American journal of human genetics. 2013; 93(1):42-53. Epub 2013/ 06/19. https://doi.org/10.1016/j.ajhg.2013.05.010 PMID: 23768515; PubMed Central PMCID: PMC3710762.

35. Feng S, Liu D, Zhan X, Wing MK, Abecasis GR. RAREMETAL: fast and powerful meta-analysis for rare variants. Bioinformatics. 2014; 30(19):2828-9. Epub 2014/06/05. https://doi.org/10.1093/ bioinformatics/btu367 PMID: 24894501; PubMed Central PMCID: PMC4173011.

36. Yang J, Ferreira T, Morris AP, Medland SE, Genetic Investigation of ATC, Replication DIG, et al. Conditional and joint multiple-SNP analysis of GWAS summary statistics identifies additional variants influencing complex traits. Nature genetics. 2012; 44(4):369-75, S1-3. Epub 2012/03/20. https://doi.org/10. 1038/ng.2213 PMID: 22426310; PubMed Central PMCID: PMC3593158.

37. Buchanan CC, Torstenson ES, Bush WS, Ritchie MD. A comparison of cataloged variation between International HapMap Consortium and 1000 Genomes Project data. J Am Med Inform Assoc. 2012; 19 (2):289-94. Epub 2012/02/10. https://doi.org/10.1136/amiajnl-2011-000652 PMID: 22319179; PubMed Central PMCID: PMC3277631.

38. Das S, Forer L, Schonherr S, Sidore C, Locke AE, Kwong A, et al. Next-generation genotype imputation service and methods. Nature genetics. 2016; 48(10):1284-7. Epub 2016/08/30. https://doi.org/10. 1038/ng.3656 PMID: 27571263; PubMed Central PMCID: PMC5157836.

39. De Jager PL, Srivastava G, Lunnon K, Burgess J, Schalkwyk LC, Yu L, et al. Alzheimer's disease: early alterations in brain DNA methylation at ANK1, BIN1, RHBDF2 and other loci. Nat Neurosci. 2014; 17 (9):1156-63. Epub 2014/08/19. https://doi.org/10.1038/nn.3786 PMID: 25129075; PubMed Central PMCID: PMC4292795.

40. De Jager PL, Shulman JM, Chibnik LB, Keenan BT, Raj T, Wilson RS, et al. A genome-wide scan for common variants affecting the rate of age-related cognitive decline. Neurobiol Aging. 2012; 33(5):1017 e1-15. Epub 2011/11/08. https://doi.org/10.1016/j.neurobiolaging.2011.09.033 PMID: 22054870; PubMed Central PMCID: PMC3307898. 
41. Wu L, Shi W, Long J, Guo X, Michailidou K, Beesley J, et al. A transcriptome-wide association study of 229,000 women identifies new candidate susceptibility genes for breast cancer. Nature genetics. 2018; 50(7):968-78. Epub 2018/06/20. https://doi.org/10.1038/s41588-018-0132-x PMID: 29915430; PubMed Central PMCID: PMC6314198.

42. Rödel E. Fisher, R. A.: Statistical Methods for Research Workers, 14. Aufl., Oliver \& Boyd, Edinburgh, London 1970. XIII, 362 S., 12 Abb., 74 Tab., 40 s. Biometrische Zeitschrift. 1971;13(6):429-30. https:// doi.org/10.1002/bimj.19710130623

43. Jansen IE, Savage JE, Watanabe K, Bryois J, Williams DM, Steinberg S, et al. Genome-wide metaanalysis identifies new loci and functional pathways influencing Alzheimer's disease risk. Nature genetics. 2019; 51(3):404-13. Epub 2019/01/09. https://doi.org/10.1038/s41588-018-0311-9 PMID: 30617256.

44. Marioni RE, Harris SE, Zhang Q, McRae AF, Hagenaars SP, Hill WD, et al. GWAS on family history of Alzheimer's disease. https://doi.org/10.1038/s41398-018-0150-6 PMID: 29777097. 2018; 8(1):99. Epub 2018/05/20. PubMed Central PMCID: PMC5959890.

45. Andaleon A, Mogil LS, Wheeler HE. Genetically regulated gene expression underlies lipid traits in Hispanic cohorts. PLoS One. 2019; 14(8):e0220827. Epub 2019/08/09. https://doi.org/10.1371/journal. pone.0220827 PMID: 31393916; PubMed Central PMCID: PMC6687110.

46. Zhu Z, Zheng Z, Zhang F, Wu Y, Trzaskowski M, Maier R, et al. Causal associations between risk factors and common diseases inferred from GWAS summary data. Nat Commun. 2018; 9(1):224. Epub 2018/01/18. https://doi.org/10.1038/s41467-017-02317-2 PMID: 29335400; PubMed Central PMCID: PMC5768719.

47. Tripathi S, Christie KR, Balakrishnan R, Huntley R, Hill DP, Thommesen L, et al. Gene Ontology annotation of sequence-specific DNA binding transcription factors: setting the stage for a large-scale curation effort. Database (Oxford). 2013; 2013:bat062. Epub 2013/08/29. https://doi.org/10.1093/database/ bat062 PMID: 23981286; PubMed Central PMCID: PMC3753819.

48. Chiba-Falek O, Gottschalk WK, Lutz MW. The effects of the TOMM40 poly-T alleles on Alzheimer's disease phenotypes. Alzheimers Dement. 2018; 14(5):692-8. Epub 2018/03/11. https://doi.org/10.1016/j. jalz.2018.01.015 PMID: 29524426; PubMed Central PMCID: PMC5938113.

49. Xi ZQ, Sun JJ, Wang XF, Li MW, Liu XZ, Wang LY, et al. HSPBAP1 is found extensively in the anterior temporal neocortex of patients with intractable epilepsy. Synapse. 2007; 61(9):741-7. Epub 2007/06/ 15. https://doi.org/10.1002/syn.20417 PMID: 17568411.

50. Albers MW, Gilmore GC, Kaye J, Murphy C, Wingfield A, Bennett DA, et al. At the interface of sensory and motor dysfunctions and Alzheimer's disease. Alzheimers Dement. 2015; 11(1):70-98. Epub 2014/ 07/16. https://doi.org/10.1016/j.jalz.2014.04.514 PMID: 25022540; PubMed Central PMCID: PMC4287457.

51. Saha $P$, Sen N. Tauopathy: A common mechanism for neurodegeneration and brain aging. Mech Ageing Dev. 2019; 178:72-9. Epub 2019/01/23. https://doi.org/10.1016/j.mad.2019.01.007 PMID: 30668956; PubMed Central PMCID: PMC6377302.

52. Sherva R, Tripodis Y, Bennett DA, Chibnik LB, Crane PK, de Jager PL, et al. Genome-wide association study of the rate of cognitive decline in Alzheimer's disease. Alzheimer's \& dementia: the journal of the Alzheimer's Association. 2014; 10(1):45-52. Epub 2013/03/25. https://doi.org/10.1016/j.jalz.2013.01. 008 PMID: 23535033.

53. Kamboh MI, Fan KH, Yan Q, Beer JC, Snitz BE, Wang X, et al. Population-based genome-wide association study of cognitive decline in older adults free of dementia: identification of a novel locus for the attention domain. Neurobiol Aging. 2019; 84:239.e15-.e24. Epub 2019/04/08. https://doi.org/10.1016/j. neurobiolaging.2019.02.024 PMID: 30954325; PubMed Central PMCID: PMC6739197.

54. Beecham GW, Hamilton K, Naj AC, Martin ER, Huentelman M, Myers AJ, et al. Genome-wide association meta-analysis of neuropathologic features of Alzheimer's disease and related dementias. PLoS Genet. 2014; 10(9):e1004606. Epub 2014/09/05. https://doi.org/10.1371/journal.pgen.1004606 PMID: 25188341 ; PubMed Central PMCID: PMC4154667.

55. Hu X, Pickering EH, Hall SK, Naik S, Liu YC, Soares H, et al. Genome-wide association study identifies multiple novel loci associated with disease progression in subjects with mild cognitive impairment. TransI Psychiatry. 2011; 1(11):e54. Epub 2011/01/01. https://doi.org/10.1038/tp.2011.50 PMID: 22833209; PubMed Central PMCID: PMC3309471.

56. Sandí M-J, Marshall CB, Balan M, Coyaud É, Zhou M, Monson DM, et al. MARK3-mediated phosphorylation of ARHGEF2 couples microtubules to the actin cytoskeleton to establish cell polarity. Science Signaling. 2017; 10(503):eaan3286. https://doi.org/10.1126/scisignal.aan3286 PMID: 29089450

57. Sun D, Yu Z, Fang X, Liu M, Pu Y, Shao Q, et al. LncRNA GAS5 inhibits microglial M2 polarization and exacerbates demyelination. EMBO Rep. 2017; 18(10):1801-16. Epub 2017/08/14. https://doi.org/10. 15252/embr.201643668 PMID: 28808113. 
58. Sun Z, Xu Y. Nuclear Receptor Coactivators (NCOAs) and Corepressors (NCORs) in the Brain. Endocrinology. 2020; 161(8). https://doi.org/10.1210/endocr/bqaa083 PMID: 32449767

59. Yang R, Zhan M, Guo M, Yuan H, Wang Y, Zhang Y, et al. Yolk sac-derived Pdcd11-positive cells modulate zebrafish microglia differentiation through the NF-kB-Tgf $\beta 1$ pathway. Cell Death \& Differentiation. 2020. https://doi.org/10.1038/s41418-020-0591-3 PMID: 32709934

60. Lucatelli JF, Barros AC, Silva VK, Machado Fda S, Constantin PC, Dias AA, et al. Genetic influences on Alzheimer's disease: evidence of interactions between the genes APOE, APOC1 and ACE in a sample population from the South of Brazil. Neurochem Res. 2011; 36(8):1533-9. Epub 2011/05/03. https:// doi.org/10.1007/s11064-011-0481-7 PMID: 21533863.

61. García-Ayllón M-S, Small DH, Avila J, Sáez-Valero J. Revisiting the Role of Acetylcholinesterase in Alzheimer's Disease: Cross-Talk with P-tau and $\beta$-Amyloid. Front Mol Neurosci. 2011; 4:22-. https://doi. org/10.3389/fnmol.2011.00022 PMID: 21949503.

62. Sheffield LG, Miskiewicz HB, Tannenbaum LB, Mirra SS. Nuclear pore complex proteins in Alzheimer disease. J Neuropathol Exp Neurol. 2006; 65(1):45-54. Epub 2006/01/18. https://doi.org/10.1097/01. jnen.0000195939.40410.08 PMID: 16410748.

63. Reinikainen KJ, Riekkinen PJ, Halonen T, Laakso M. Decreased muscarinic receptor binding in cerebral cortex and hippocampus in alzheimer's disease. Life Sciences. 1987; 41(4):453-61. https://doi.org/ 10.1016/0024-3205(87)90221-9 PMID: 3600187

64. Ouellette AR, Neuner SM, Dumitrescu L, Anderson LC, Gatti DM, Mahoney ER, et al. Cross-Species Analyses Identify Dlgap2 as a Regulator of Age-Related Cognitive Decline and Alzheimer's Dementia. Cell Reports. 2020; 32(9):108091. https://doi.org/10.1016/j.celrep.2020.108091 PMID: 32877673

65. Ramos-García NA, Orozco-Ibarra M, Estudillo E, Elizondo G, Gómez Apo E, Chávez Macías LG, et al. Aryl Hydrocarbon Receptor in Post-Mortem Hippocampus and in Serum from Young, Elder, and Alzheimer's Patients. Int J Mol Sci. 2020; 21(6):1983. https://doi.org/10.3390/ijms21061983 PMID: 32183254

66. Gong CX, Singh TJ, Grundke-lqbal I, Iqbal K. Phosphoprotein phosphatase activities in Alzheimer disease brain. J Neurochem. 1993; 61(3):921-7. Epub 1993/09/01. https://doi.org/10.1111/j.1471-4159. 1993.tb03603.x PMID: 8395566.

67. Remnestål J. Expression and distribution of transcription factors NPAS3 och RFX3 in Alzheimer's disease [Student thesis]2015.

68. Colton CA, Vitek MP, Wink DA, Xu Q, Cantillana V, Previti ML, et al. NO synthase 2 (NOS2) deletion promotes multiple pathologies in a mouse model of Alzheimer's disease. Proceedings of the National Academy of Sciences. 2006; 103(34):12867-72. https://doi.org/10.1073/pnas.0601075103 PMID: 16908860

69. Rusilowicz-Jones E, Jardine J, Kallinos A, Pinto-Fernandez A, Guenther F, Giurrandino M, et al. A novel USP30 inhibitor recapitulates genetic loss of USP30 and sets the trigger for PINK1-PARKIN amplification of mitochondrial ubiquitylation. bioRxiv. 2020:2020.04.16.044206. https://doi.org/10.1101/ 2020.04.16.044206

70. Myers S, Yuan H, Kang J, Tan F, Traynelis S, Low C. Distinct roles of GRIN2A and GRIN2B variants in neurological conditions [version 1; peer review: 2 approved]. F1000Research. 2019; 8(1940). https:// doi.org/10.12688/f1000research.18949.1 PMID: 31807283

71. Babenko VN, Smagin DA, Galyamina AG, Kovalenko IL, Kudryavtseva NN. Altered SIc25 family gene expression as markers of mitochondrial dysfunction in brain regions under experimental mixed anxiety/ depression-like disorder. BMC Neurosci. 2018; 19(1):79. Epub 2018/12/13. https://doi.org/10.1186/ s12868-018-0480-6 PMID: 30537945; PubMed Central PMCID: PMC6288882.

72. Yokoyama JS, Wang Y, Schork AJ, Thompson WK, Karch CM, Cruchaga C, et al. Association Between Genetic Traits for Immune-Mediated Diseases and Alzheimer Disease. JAMA Neurol. 2016; 73(6):6917. https://doi.org/10.1001/jamaneurol.2016.0150 PMID: 27088644.

73. Russ J. Systematic interaction mapping reveals novel modifiers of neurodegenerative disease processes 2012.

74. Wostyn P, Audenaert K, De Deyn PP. Choroidal Proteins Involved in Cerebrospinal Fluid Production may be Potential Drug Targets for Alzheimer's Disease Therapy. Perspect Medicin Chem. 2011; 5:117. https://doi.org/10.4137/PMC.S6509 PMID: 21487536

75. La Piana R, Weraarpachai W, Ospina LH, Tetreault M, Majewski J, Bruce Pike G, et al. Identification and functional characterization of a novel MTFMT mutation associated with selective vulnerability of the visual pathway and a mild neurological phenotype. neurogenetics. 2017; 18(2):97-103. https://doi.org/ 10.1007/s10048-016-0506-0 PMID: 28058511

76. Jeromin A, Lasseter HC, Provost AC, Daskalakis NP, Etkin A, Gehrman P, et al. Driving Progress in Posttraumatic Stress Disorder Biomarkers. Biol Psychiatry. 2020; 87(6):e13-e4. Epub 2019/10/19. https://doi.org/10.1016/j.biopsych.2019.07.036 PMID: 31623824. 
77. Mogil LS, Andaleon A, Badalamenti A, Dickinson SP, Guo X, Rotter Jl, et al. Genetic architecture of gene expression traits across diverse populations. PLOS Genetics. 2018; 14(8):e1007586. https://doi. org/10.1371/journal.pgen.1007586 PMID: 30096133

78. Chen H, Wang C, Conomos MP, Stilp AM, Li Z, Sofer T, et al. Control for Population Structure and Relatedness for Binary Traits in Genetic Association Studies via Logistic Mixed Models. American journal of human genetics. 2016; 98(4):653-66. Epub 2016/03/29. https://doi.org/10.1016/j.ajhg.2016.02. 012 PMID: 27018471; PubMed Central PMCID: PMC4833218. 